\title{
Vertex Operators for Non-Simply-Laced Algebras
}

\author{
P. Goddard ${ }^{1}$, W. Nahm ${ }^{2}$, D. Olive ${ }^{3}$, and A. Schwimmer ${ }^{4 \star}$ \\ ${ }^{1}$ Department of Applied Mathematics and Theoretical Physics, University of Cambridge, \\ Silver Street, Cambridge CB3 9EW, United Kingdom \\ ${ }^{2}$ Physikalisches Institut, Universität Bonn, Nussallee 12, D-5300 Bonn 1, \\ Federal Republic of Germany \\ ${ }^{3}$ Blackett Laboratory, Imperial College, London SW7 2BZ, U.K. \\ ${ }^{4}$ Laboratoire de Physique Theorique de L'Ecole Normale Superieure, 24 Rue Lhomond, \\ F-75231 Paris Cedex 05, France
}

\begin{abstract}
A vertex operator construction is given for the level one representations of the affine Kac-Moody algebras associated with non-simply-laced finite-dimensional Lie algebras, using free boson and interacting fermion fields. The fermion fields are constructed explicitly and a detailed discussion is given of the theory of the cocycles necessary for this and other vertex operator constructions. The construction is related in detail to the folding of Dynkin diagrams and a generalisation of it yields Freudenthal's magic square.
\end{abstract}

\section{Introduction}

Since Frenkel and Kac [1] and, independently, Segal [2] constructed level one represenations of untwisted affine Kac-Moody algebras $\hat{g}$, associated with simple Lie algebras $g$ which are simply-laced, using the vertex operators of string theory, a corresponding construction for the non-simply-laced case has been sought. The cases where $g$ is simply laced, i.e. all roots have a common length, are $g=\mathrm{su}(r+1)$, so $(2 r), E_{6}, E_{7}$, and $E_{8}$, and the non-simply laced cases are $g=\operatorname{so}(2 r+1), \operatorname{sp}(r)$, each if $r \geqq 2, G_{2}$ and $F_{4}$. (For a review of terminology and results on Kac-Moody and Virasoro algebras, developed in relation to their applications in quantum physics; see [3].) In this paper we provide a vertex operator construction for the non-simply laced case.

There are a number of reasons for wanting such a construction. For example, it gives us a more uniform approach to the most basic representations of all the untwisted algebras $\hat{g}$. It involves the introduction of fermion operators associated with the short roots. The Frenkel-Kac-Segal (FKS) construction enables one to build non-abelian internal symmetries into string theories in an intrinsic way by compactifying some of the dimensions in which the string moves to form the maximal torus of a simply-laced group [4]. This has been exploited to construct potentially realistic unified string theories of particle interactions [5]. These theories involve fermions and the present generalisation of the FKS construction by the

\footnotetext{
* On leave from the Weizmann Institute, Israel
} 
introduction of fermions produces a greater resemblance with string theories of physical interest. It may lead to new physical possibilities and it may be relevant to understanding further the structure of existing theories.

If $g$ has totally antisymmetric structure constants $f^{a b c}$, the corresponding untwisted affine Kac-Moody algebra $\hat{g}$ is defined by the commutation relations

$$
\left[T_{m}^{a}, T_{n}^{b}\right]=\text { if }{ }^{a b c} T_{m+n}^{c}+k m \delta^{a b} \delta_{m,-n},
$$

where $m, n \in \mathbb{Z}$, the set of integers, and $a, b, c$ run over the values 1 to $\operatorname{dim} g ; k$ is a central term taking a numerical value in any irreducible unitary representation of $\hat{g}$. In such a representation $x=2 k / \psi^{2}$, where $\psi$ is the long root of $g$, is a positive integer called the level of the representation. Information about possible vertex operator representations of $\hat{g}$ can be obtained by considering the Virasoro algebra

$$
\left[\mathscr{L}_{M}, \mathscr{L}_{N}\right]=(M-N) \mathscr{L}_{M+N}+\frac{c}{12} M\left(M^{2}-1\right) \delta_{M,-N^{\prime}}
$$

associated with $\hat{g}$, given by the Sugawara construction,

$$
\mathscr{L}_{N}=\frac{1}{\beta} \sum_{m} \sum_{a} \underset{\times}{\times} T_{m+N}^{a} T_{-m}^{a} \stackrel{\times}{\times},
$$

where the sum is over $m \in \mathbb{Z}, 1 \leqq a \leqq \operatorname{dim} g, \beta=2 k+Q, Q$ being the value of quadratic Casimir operator for $g$ in the adjoint representation, and the crosses denote normal ordering with respect to the $T_{n}^{a}$ (see e.g. [3] for further details).

In (1.2) $c$ takes the value

$$
c_{g}=\frac{x \operatorname{dim} g}{x+\tilde{h}(g)}
$$

where $\tilde{h}(g)=Q / \psi^{2}$ is the dual Coxeter number. This can be written in the form

$$
\tilde{h}(g)=\frac{n_{L}}{r}+\frac{n_{S}}{r}\left(\frac{S}{L}\right)^{2},
$$

where $r$ is the rank of $g$ and $n_{L}, n_{S}$ are the number of long roots (length $L$ ) and short roots (length $S$ ) of $g$, respectively. Thus, since $\operatorname{dim} g=n_{L}+n_{S}+r$,

$$
c_{g}=\frac{n_{L}[x-1]+n_{S}\left[x-(S / L)^{2}\right]}{x+h}+r .
$$

If we have a vertex operator representation of $\hat{g}$ in a Fock space generated by $n_{B}$ (independent) boson fields and $n_{F}$ (independent) fermi fields, we have a Virasoro algebra $L_{N}$ satisfying (1.2) with $c$ equal to

$$
c^{\prime}=n_{B}+\frac{1}{2} n_{F}
$$

Further [6], since

$$
\left[L_{M}, T_{N}^{a}\right]=\left[\mathscr{L}_{M}, T_{N}^{a}\right]=-N T_{M+N}^{a},
$$

$K_{M}=L_{M}-\mathscr{L}_{M}$ defines a Virasoro algebra, commuting with $\mathscr{L}_{M}$, with $c=c^{\prime}-c_{g}$. It can be shown that this must not be negative. Hence

$$
c^{\prime} \geqq c_{g}
$$


The FKS construction provides a level 1 representation of a simply-laced $g$ with $r=$ rank $g$ boson fields. It follows from (1.6) and (1.9) that if either $x>1$ or $g$ is not simply laced, so that $S^{2} / L^{2}<1$, we need more boson or fermion fields.

A non-simply laced algebra $g$ contains a simply laced subalgebra $g_{L}$, of the same rank $r$, generated by the Cartan subalgebra of $g$ together with step operators corresponding to the long roots of $g$. The level 1 representation of $\hat{g}$ splits up into level 1 representations of $\hat{g}_{L}$, which can be constructed, using the KFS construction, with $r$ independent boson fields. If we wish to extend this representation of $\hat{g}_{L}$, by the addition of $n_{F}$ independent fermion fields we shall need

$$
\begin{gathered}
n_{F} \geqq \frac{n_{S}}{(h+1)}, \\
\frac{2 n(n+1)}{n+3},
\end{gathered}
$$

where $n=1$ for $\operatorname{so}(2 r+1), n=r+1), n=r-1$ for $\operatorname{sp}(r), n=2$ for $F_{4}$. ( $n$ is the number of short simple roots of $g$.) To get an irreducible (or finitely reducible) representation in the whole Fock space generated by $r$ boson fields and $n_{F}$ fermion fields we would need (1.10) to be an equality; but typically (1.11) is not an integer, the exceptions being $n=1,3$ and 9 . This shows that vertex operators for a nonsimply laced $g$ cannot in general be constructed from independent fermions, or rather, that, if this can be done, there must be some parts of the fields which are not used.

In our construction we shall find that we need $\frac{1}{2} n(n+1)$ real fermion fields, but that these are fermions which interact with each other in some sense. We construct them using $n$ independent boson fields, but the fermion fields operate in a subspace of the corresponding bosonic Hilbert space. We can construct a Virasoro algebra, $L_{N}^{F}$, out of these fermions and this has $c$ given by $n(n+1) /(n+3)$. The Virasoro algebra, $L_{N}^{B}$, for all the bosons out of which the fermions are made, has $c=n$ and so the difference, $\mathscr{K}_{N}=L_{N}^{B}-L_{N}^{F}$, which commutes with the fermion fields, has $c=2 n /(n+3)$. The subspace in which the fermions act can be defined by conditions

$$
\mathscr{K}_{N}|\phi\rangle=0, N>0 ; \mathscr{K}_{0}|\phi\rangle=\lambda|\phi\rangle .
$$

For $n=1,2$, we have $c<1$ and so, using the results of Friedan, Qiu and Shenker (FQS) [7], and an argument in [6], in these cases there are only finitely many states satisfying (1.12). It follows that the whole representation space is finitely-reducible under the action of the Kac-Moody algebra and the Virasoro algebra, $\mathscr{K}_{N}$.

Our construction is described in the next five sections of this paper whose plan is as follows. In Sect. 2 we review the vertex operator construction for simply-laced groups, partly for the purpose of establishing notation. In Sect. 3 we review properties of the fermionic operators associated with points of unit length and we show how, with one extra fermion field, we can extend the FKS construction for so $(2 r)$ to obtain a vertex operator construction for so $(2 r+1)$. Next, in Sect. 4 , restricting our attention to algebras for which $L^{2} / S^{2}=2$, we consider the general structure of the extra fermion fields that we need to introduce to multiply the vertex operators associated with short roots, taken to have unit length. We find we need a different real fermion for each orbit of the short roots of $g$ under the Weyl group of 
$g_{L}$. Having established the general requirements for these new fermi fields, it is necessary to consider more carefully the functions of momentum (cocycles) needed to correct for the anomalous signs in commutators; Secttion 5 is devoted to an account of the theory of their construction [1]. Our construction involves in an essential way cocycles taking the values $\pm i$ as well as \pm 1 , and, for convenience, other roots of unity. In Sect. 6, we explain how to construct the interacting fermions, showing that the necessary cocycles can be found consistently.

The remaining sections of the paper deal with other aspects of the construction and its generalisations. A non-simply laced $g$ can be embedded in a larger simply laced $g_{0}$ in such a way that the common root length of $g_{0}$ is that of the long roots of $g$. This implies that we can obtain a level one representation of $\hat{g}$ from a level one of representation of $\hat{g}_{0}$. We show how our construction can be obtained from this embedding in Sect. 7. In Sect. 8, we show how the construction works for the special case of $G_{2}$. We generalise the construction in Sect. 9 in a way that leads to the magic square of Freudenthal. The main guide in our discussion was the Sugawara construction of the Virasoro algebra, and this is considered further in Sect. 10, where we show that we do indeed obtain the appropriate $c$-numbers. In Sect. 11 we conclude with speculations on the connection of this work with fermionic string theory and a comment on the bosonic realisation of the discrete series of unitary representations of the Virasoro algebra.

\section{Vertex Operator Construction for Simply-Laced Algebras}

In this section we shall review briefly the construction of the vertex operator representation for simply-laced algebras.

The root system of a compact Lie algebra $g$ of rank $r$ consists of a set $\Phi$ of real $r$-dimensional vectors with the properties:

$$
\begin{aligned}
& \text { if } \alpha \in \Phi \text {, then } \lambda \alpha \in \Phi \text { if and only if } \lambda= \pm 1 \text {; } \\
& \qquad \text { if } \alpha, \beta \in \Phi \text {, then } 2 \alpha \cdot \beta / \alpha^{2} \in \mathbb{Z} \text {; } \\
& \text { if } \alpha, \beta \in \Phi \text {, then } \sigma_{\alpha}(\beta) \equiv \beta-\left(2 \alpha \cdot \beta / \alpha^{2}\right) \alpha \in \Phi .
\end{aligned}
$$

The linear transformation $\sigma_{\alpha}$ is called a Weyl transformation and the $\sigma_{\alpha}, \alpha \in \Phi$, generate a finite group, $W(g)$, the Weyl group of $g$. The root lattice of $g, \Lambda_{R}(g)$, is the lattice generated by the $\alpha \in \Phi$; and the weight lattice

$$
\Lambda_{W}(g)=\left\{\mu: 2 \alpha \cdot \mu / \alpha^{2} \in \mathbb{Z} \quad \text { for all } \quad \alpha \in \Phi\right\} .
$$

Clearly $\Lambda_{W}(g) \supset \Lambda_{R}(g)$. If $g$ is simply-laced, i.e. $\alpha^{2}$ is the same for all $\alpha \in \Phi$, we can choose the normalisation of our scalar product so that $\alpha^{2}=2$ for each $\alpha$. In this case $\Lambda_{W}(g)=\Lambda_{R}(g)^{*}$, the lattice reciprocal to $\Lambda_{R}(g)$.

The vertex operator representation of a simply-laced $g$ is defined in a Hilbert space $\mathscr{H}$, generated by operators $\alpha_{n}^{i}, n \in \mathbb{Z}, 1 \leqq i \leqq r$, satisfying the commutation relations

$$
\left[\alpha_{m}^{i}, \alpha_{n}^{j}\right]=m \delta^{i j} \delta_{m,-n},
$$

$1 \leqq i, j \leqq r, m, n \in \mathbb{Z}$, and the hermiticity conditions

$$
\alpha_{m}^{i \dagger}=\alpha_{-m}^{i}
$$


from states $|\lambda\rangle, \lambda \in \Lambda$, obeying

$$
\begin{aligned}
p^{i}|\lambda\rangle & =\lambda^{i}|\lambda\rangle, \\
\alpha_{m}^{i}|\lambda\rangle & =0, m>0, \\
\left\langle\lambda^{\prime} \mid \lambda\right\rangle & =\delta_{\lambda \lambda^{\prime}},
\end{aligned}
$$

where $p^{\mu} \equiv \alpha_{0}^{\mu}$. Here $\Lambda$ is an $r$-dimensional lattice with $\Lambda_{W}(g) \supset \Lambda \supset \Lambda_{R}(g)$. In this context, we cannot define a position operator $q^{i}$, but we can define $e^{i \lambda \cdot q}$ for $\lambda \in \Lambda$ by

$$
e^{i \lambda \cdot q}\left|\lambda^{\prime}\right\rangle=\left|\lambda+\lambda^{\prime}\right\rangle
$$

together with the condition it commutes with the $\alpha_{n}^{j}, n \neq 0$. The whole of $\mathscr{H}$ is generated from the vacuum vector $|0\rangle$ by $\alpha_{n}^{i}, 1 \leqq i \leqq r, n \in \mathbb{Z}$ and $e^{i \lambda \cdot q}, \lambda \in \Lambda$.

We introduce vertex operators, defined by

$$
U(\alpha, z) \equiv z^{\alpha^{2} / 2}: \exp \{i \alpha \cdot Q(z)\}:
$$

where

$$
Q^{j}(z)=q^{j}-i p^{j} \log z+i \sum_{n \neq 0} \frac{\alpha_{n}^{j}}{n} z^{-n},
$$

and the colons denote normal ordering with respect to the $\alpha_{n}^{j}$; that is $\alpha_{n}^{j}, n>0$, is moved to the right of $\alpha_{m}^{i}, m<0$, and $p^{j}$ to the right of $q^{i}$. Thus

$$
U(\alpha, z)=\exp \left\{i \alpha \cdot Q_{<}(z)\right\} \exp \left\{i \alpha \cdot Q_{0}(z)\right\} \exp \left\{i \alpha \cdot Q_{>}(z)\right\},
$$

where

$$
Q_{>}^{j}(z)=i \sum_{n>0} \frac{\alpha_{n}^{j}}{n} z^{-n}, \quad Q_{<}^{j}(z)=i \sum_{n<0} \frac{\alpha_{n}^{j}}{n} z^{-n}
$$

and

$$
Q_{0}^{j}(z)=q^{j}-i p^{j} \log z
$$

so that

$$
e^{i \alpha \cdot Q_{0}(z)}=z^{\frac{\alpha^{2}}{2}}: e^{i \alpha \cdot Q_{0}(z)}:=z^{\frac{\alpha^{2}}{2}} e^{i \alpha \cdot q} z^{\alpha \cdot p} .
$$

The vertex operator $U(\alpha, z)$ is a single-valued function of $z$ if $\alpha \in \Phi$ and $p \in \Lambda$. Thus we can define

$$
A_{\alpha}=\frac{1}{2 \pi i} \oint U(\alpha, z) \frac{d z}{z},
$$

where the integration contour encircles the origin once positively. To attempt to calculate the algebra of the $A_{\alpha}$ we use

$$
\begin{aligned}
& \exp \left\{i \alpha \cdot Q_{>}(z)\right\} \exp \left\{i \beta \cdot Q_{<}(\zeta)\right\} \\
& \quad=(1-\zeta / z)^{\alpha \cdot \beta} \exp \left\{i \beta \cdot Q_{<}(\zeta)\right\} \exp \left\{i \alpha \cdot Q_{>}(z)\right\} \text { if }|\zeta|<|z|,
\end{aligned}
$$

which follows from

$$
\left[Q_{>}^{i}(z), Q_{<}^{j}(\zeta)\right]=\delta^{i j} \log (1-\zeta / z)
$$


This enables us to normal order

$$
\begin{aligned}
U(\alpha, z) U(\beta, \zeta) & =(z-\zeta)^{\alpha \cdot \beta}: U(\alpha, z) U(\beta, \zeta): \text { for }|\zeta|<|z|, \\
& \equiv U_{\alpha, \beta}(z, \zeta) .
\end{aligned}
$$

In the case we are considering, $\alpha^{2}=\beta^{2}=2$. The right-hand side of Eq. (2.16) is a regular function of $z, \zeta$ except for $z=0, \zeta=0$ and $z=\zeta$. If we calculate the product in the other order we obtain the same expression as a function of $z$ and $\zeta$, apart from a factor of $(-1)^{\alpha \cdot \beta}$, which could be either +1 or -1 , this time valid if $|\zeta|>|z|$. Thus what we can easily evaluate is

$$
\begin{aligned}
A_{\alpha} A_{\beta}-(-1)^{\alpha \cdot \beta} A_{\beta} A_{\alpha} & =\frac{1}{(2 \pi i)^{2}}\left\{\oint_{|\zeta|<|z|} \frac{d \zeta}{\zeta} \oint \frac{d z}{z}-\oint_{|\zeta|>|z|} \frac{d \zeta}{\zeta} \oint \frac{d z}{z}\right\} U_{\alpha, \beta}(z, \zeta) \\
& =\frac{1}{(2 \pi i)^{2}} \oint_{0} \frac{d \zeta}{\zeta} \oint_{\zeta} \frac{d z}{z} U_{\alpha, \beta}(z, \zeta),
\end{aligned}
$$

where the $z$ contour encircles $\zeta$ positively once and the $\zeta$ contour encircles the origin positively once.

If $\alpha, \beta \in \Phi$, the possible values of $\alpha \cdot \beta$ are $\pm 2, \pm 1,0$, since $\alpha \cdot \beta \in \mathbb{Z}$ and $\alpha^{2}=\beta^{2}=2$. If $\alpha \cdot \beta \geqq 0$, the right-hand side of (2.17) vanishes because in this case $U_{\alpha, \beta}(z, \zeta)$ is regular at $z=\zeta$. If $\alpha \cdot \beta=-1, \alpha+\beta$ is a root and this is the only case in which this happens. Then $U_{\alpha, \beta}(z, \zeta)$ has a simple pole at $z=\zeta$ and we obtain $A_{\alpha+\beta}$. If $\alpha \cdot \beta=-2$, then $\alpha=-\beta$ and we have a double pole at $z=\zeta$; in this case we obtain $\alpha \cdot p$. Thus we have obtained the following results:

$$
\begin{aligned}
& A_{a} A_{\beta}-(-1)^{\alpha \cdot \beta} A_{\beta} A_{\alpha}=0 \quad \text { if } \quad \alpha \cdot \beta \geqq 0 \text {, } \\
& =A_{\alpha+\beta} \text { if } \alpha \cdot \beta=-1 \text {, } \\
& =\alpha \cdot p \quad \text { if } \quad \alpha=-\beta \text {. }
\end{aligned}
$$

Additionally we have that

$$
\left[p^{i}, A_{\alpha}\right]=\alpha^{i} A_{\alpha}
$$

To remove the annoying factor of $(-1)^{\alpha \cdot \beta}$, and obtain a representation of $g$, we introduce, following Frenkel and $\mathrm{Kac}$ [1], a function $c_{\alpha}$ of the momentum operator $p$ for each $\alpha \in \Lambda_{R}$ with properties such that, if $\hat{c}_{\alpha}=e^{i q \cdot \alpha} c_{\alpha}$, then

$$
\begin{aligned}
\hat{c}_{\alpha} \hat{c}_{\beta} & =(-1)^{\alpha \cdot \beta} \hat{c}_{\beta} \hat{c}_{\alpha}, \\
\hat{c}_{\alpha} \hat{c}_{-\alpha} & =1, \\
\hat{c}_{\alpha} \hat{c}_{\beta} & =\varepsilon(\alpha, \beta) \hat{c}_{\alpha+\beta},
\end{aligned}
$$

where the cocycle $\varepsilon(\alpha, \beta)$ takes the values \pm 1 . Our problem is then solved because

$$
E^{\alpha}=A_{\alpha} c_{\alpha}, \alpha \in \Phi ; \quad H^{i}=p^{i}, 1 \leqq i \leqq r,
$$

satisfy the algebra $g$. The construction of $c_{\alpha}$ and $\varepsilon(\alpha, \beta)$ is considered in detail in Sect. 5 . 
To get the corresponding untwisted affine Kac-Moody algebra $\hat{g}$ we take the Laurent coefficients of

$$
U(\alpha, z) c_{\alpha}=\sum_{n \in \mathbb{Z}} E_{n}^{\alpha} z^{-n}
$$

and

$$
P^{j}(z) \equiv \sum_{n \in \mathbb{Z}} \alpha_{n}^{j} z^{-n}
$$

writing $H_{n}^{j} \equiv \alpha_{n}^{j}$ for this purpose. These then satisfy

$$
\begin{array}{rlrl}
{\left[H_{m}^{i}, H_{n}^{j}\right]} & =m \delta^{i j} \delta_{m,-n}, & \\
{\left[H_{m}^{i}, E_{n}^{\alpha}\right]} & =\alpha^{i} E_{m+n}^{\alpha}, & \\
{\left[E_{m}^{\alpha}, E_{n}^{\beta}\right]} & =\varepsilon(\alpha, \beta) E_{m+n}^{\alpha+\beta}, & & \text { if } \alpha+\beta \in \Phi, \\
& =\alpha \cdot H_{m+n}+m \delta_{m+n, 0}, & & \text { if } \alpha=-\beta, \\
& =0, & & \text { otherwise. }
\end{array}
$$

This provides a level one of representation of $\hat{g}$.

\section{Vertex Operators Associated with Points of Unit Length}

If we consider a simple Lie algebra $g$ with roots of two different lengths, the ratio of their squares being 2 , we can normalise these so that the long roots $\Phi_{L}$ have squared length 2 and the short roots $\Phi_{S}$ have unit length. (We shall discuss $G_{2}$ in Sect. 8.) It is easy to check that the long roots form the root system of a simply-laced Lie algebra $g_{L} \subset g$, and we can represent $g_{L}$, and $\hat{g}_{L}$, by vertex operators as described in Sect. 2 . Constructing a vertex operator associated with points $\lambda \in \Phi_{S}$ is not so straightforward and in this section we review results on vertex operators for points of squared length 1.

Consider again the space $\mathscr{H}$ defined in Sect. 2, built up from momentum states $|\lambda\rangle, \lambda \in \Lambda$, where now, as well as a set of points $\Phi_{L}$ of squared length $2, \Lambda$ contains a set of points $\Phi_{S}$ of squared length 1. If we associate to points $e \in \Phi_{S}$ operators $A_{e}$ defined by (2.13), and use (2.16) to try to calculate the algebra of such operators, we have

$$
\begin{aligned}
U(e, z) U(f, \zeta)= & \exp \left\{i e \cdot Q_{<}(z)+i f \cdot Q_{<}(\zeta)\right\} \\
& \cdot(z-\zeta)^{e \cdot f} z^{1 / 2} \zeta^{1 / 2} e^{i(e+f) \cdot q} z^{e \cdot p} \zeta^{f \cdot p} \\
& \cdot \exp \left\{i e \cdot Q_{>}(z)+i f \cdot Q_{>}(\zeta)\right\} \text { for }|\zeta|<|z| \\
\equiv & U_{e, f}(z, \zeta) .
\end{aligned}
$$

To be able to proceed as before we need this to be single valued about $z=\zeta$. Since $|e \cdot f| \leqq 1$, as $e^{2}=f^{2}=1$, this forces either $f= \pm e$ or $e \cdot f=0$. Further we need

$$
\exp \left\{i e \cdot Q_{0}(z)\right\}|\lambda\rangle=z^{1 / 2+e \cdot \lambda}|e+\lambda\rangle
$$

to be single-valued. Clearly this cannot be true for the whole of the lattice $\Lambda$, because it fails for $\lambda=0$, so we restrict our attention for the moment to momenta $\lambda$ in some subset $M \subset \Lambda$ for which this is true. 
With these assumptions on single-valuedness we find

$$
\begin{aligned}
A_{e}^{2} & =0, \\
{\left[A_{e}, A_{f}\right] } & =0, \quad e \cdot f=0, \\
\left\{A_{e}, A_{-e}\right\} & =1 .
\end{aligned}
$$

These equations, especially (3.3a) and (3.3c), imply that $A_{e}$ is fermionic in character. If we require $e \cdot f \in \mathbb{Z}$ for all $e, f \in \Phi_{S}$, we are effectively restricted to so $(2 n+1)$. If we also assume $e \cdot \alpha \in \mathbb{Z}$ if $e \in \Phi_{S}, \alpha \in \Phi_{L}$ we can also show, by a similar calculation, that

$$
\begin{gathered}
A_{e} A_{\alpha}-(-1)^{\alpha \cdot e} A_{\alpha} A_{e}=0, \quad \alpha \cdot e=0 \quad \text { or } \quad 1, \\
A_{e} A_{\alpha}+A_{\alpha} A_{e}=A_{e+\alpha}, \quad \alpha \cdot e=-1 .
\end{gathered}
$$

[Since $|\alpha \cdot e| \leqq \sqrt{2}$, this exhausts all the possibilities given that $e \cdot \alpha \in \mathbb{Z}$.] If $A_{\alpha}$, $\alpha \in \Phi_{L}$, is bosonic and $A_{e}, e \in \Phi_{S}$, is fermionic, we need to correct some signs again to obtain the standard set of commutation or anticommutation relations. This can be done by introducing functions $c_{x}$ of the momentum for $\mathrm{x} \in \Lambda_{R}(g)$, the lattice generated by $\Phi_{L}$ and $\Phi_{S}$, such that $\hat{c}_{x}=e^{i q \cdot x} c_{x}$ has the properties $(2.20 \mathrm{~b})$ and $(2.20 \mathrm{c})$ but with $(2.20 \mathrm{~d})$ generalised to

$$
\hat{c}_{x} \hat{c}_{y}=(-1)^{x \cdot y+x^{2} y^{2}} \hat{c}_{y} \hat{c}_{x}
$$

which is different only when $x^{2}$ and $y^{2}$ are both odd integers. Then, defining $\Psi^{e}=A_{e} c_{e}, E^{\alpha}=A_{\alpha} c_{\alpha}$, we correct for these signs. We can define an affinisation of this algebra by

$$
U(\alpha, z) c_{\alpha}=\sum_{n \in \mathbb{Z}} E_{n}^{\alpha} z^{-n}
$$

and

$$
U(e, z) c_{e}=\sum_{n \in \mathbb{Z}} \Psi_{n}^{e} z^{-n}
$$

and then Eqs. (2.24) are supplemented by

$$
\begin{aligned}
{\left[E_{n}^{\alpha}, \Psi_{n}^{e}\right] } & =\varepsilon(\alpha, e) \Psi_{m+n}^{\alpha+e}, & & \alpha+e \in \Phi_{S}, \\
& =0, & & \text { otherwise. } \\
\left\{\Psi_{m}^{e}, \Psi_{n}^{f}\right\} & =\delta_{m,-n}, & & \text { if } e=-f, \\
& =0, & & \text { otherwise. } \\
{\left[H_{M}^{i}, \Psi_{n}^{e}\right] } & =e^{i} \Psi_{m+n}^{e} . & &
\end{aligned}
$$

Since the vectors $e, f \in \Phi$ satisfy $e= \pm f$ or $e \cdot f=0$, they generate a cubic lattice $\mathbb{Z}^{r}$ and we might as well take $\Phi_{S}$ to be the set of points of unit length on this lattice and $\Phi_{L}$ to be the points of squared length 2 . Then $\Phi=\Phi_{S} \cup \Phi_{L}$ is the root system of $g=\operatorname{so}(2 r+1)$ and, if

$$
\lambda_{0}=\left(\frac{1}{2}, \frac{1}{2}, \ldots, \frac{1}{2}\right),
$$

$\Lambda_{R}(g)=\mathbb{Z}^{r}$ and $M=\mathbb{Z}^{r}+\lambda_{0}$. The weight lattice $\Lambda_{W}(g)=\mathbb{Z}^{r} \cup M$. 
The operators $\Psi_{m}^{e}$ are only defined on states with momentum in $M$, whilst the $E_{m}^{\alpha}$ are defined on all states with momentum in $\Lambda_{W}(g)$. We can define operators associated with vectors $e \in \Phi_{S}$, acting on states with momentum in $\Lambda_{R}(g)=\mathbb{Z}^{r}$ by using the fact that $U(e, z) c_{e}$ has an expansion in half-integral powers of $z$ when acting on such states. Thus we write

$$
\Psi^{e}(z) \equiv U(e, z) c_{e}=\sum_{s \in \mathbb{Z}+1 / 2} \Psi_{s}^{e} z^{-s}
$$

on states with momentum in $\Lambda_{R}(g)$. Then

$$
\begin{aligned}
\left\{\Psi_{s}^{e}, \Psi_{t}^{f}\right\} & =\delta_{s,-t}, & & \text { if } e=-f, \\
& =0, & & \text { otherwise. }
\end{aligned}
$$

The $\Psi_{m}^{e}, \Psi_{s}^{e}$ are like Ramond and Neveu-Schwarz oscillators, respectively. To make this clearer, let $e_{i} \in \Phi_{S}, 1 \leqq i \leqq r$, be an orthonormal basis for $\mathbb{Z}^{r}$, and define

$$
\begin{array}{cc}
\Psi^{j}(z)=2^{-1 / 2}\left[\Psi^{e_{j}}(z)+\Psi^{-e_{j}}(z)\right], & 1 \leqq j \leqq r, \\
\Psi^{r+j}(z)=2^{-1 / 2} i\left[\Psi^{e_{j}}(z)-\Psi-e_{j}(z)\right], & 1 \leqq j \leqq r .
\end{array}
$$

Then expanding

$$
\Psi^{j}(z)=\sum_{s \in \mathbb{Z}+1 / 2} b_{s}^{j} z^{-s} \quad \text { or } \quad \sum_{n \in \mathbb{Z}} d_{n}^{j} z^{-n}, \quad 1 \leqq j \leqq 2 r,
$$

depending on whether it is acting on states with momentum in $\mathbb{Z}^{r}$ or $M$, we have

$$
\begin{gathered}
\left\{b_{s}^{i}, b_{t}^{j}\right\}=\delta^{i j} \delta_{s,-t}, \\
\left\{d_{m}^{i}, d_{n}^{j}\right\}=\delta^{i j} \delta_{m,-n}, \\
b_{s}^{i \dagger}=b_{-s}^{i} \text { and } d_{m}^{i \dagger}=d_{-m}^{i} .
\end{gathered}
$$

The operators $E_{m}^{\alpha}, \alpha \in \Phi_{L}, H_{n}^{i}, 1 \leqq i \leqq r$, provide a level 1 representation of the (untwisted) affine version of $g_{L}=$ so $(2 r)$. This cannot be extended to a level 1 representation of the affine algebra associated with $g=$ so $(2 r+1)$ because the central term for the Sugawara construction of the Virasoro algebra for this representation of $\hat{g}$ is $r+\frac{1}{2}$, indicating that we need at least $r$ bosons and one fermion field, independent of one another; thus, since we already have $r$ boson fields, we need an extra fermion. We can see how to introcuce this if we consider the way we can rewrite our representation of so $(2 r)$ in terms of bilinears in fermions:

$$
\begin{gathered}
E^{e_{i}+e_{j}}(z)=\Psi^{e_{i}}(z) \Psi^{e_{j}}(z), \quad i \neq j, \\
e_{j} \cdot H(z)={ }_{\circ}^{\circ} \Psi^{e_{j}}(z) \Psi-e_{j}(z)_{\circ}^{\circ},
\end{gathered}
$$

where the open dots denote normal ordering with respect to the fermionic oscillators $b_{s}^{i}$ or $d_{m}^{j}$. [This is not necessary in (3.18) because $\Psi^{e_{i}}, \Psi^{e_{j}}$, anticommute for $i \neq j$.] The $\Psi^{e_{i}}(z)$ are Ramond fields (R) acting on states with momentum in $M$ and Neveu-Schwarz fields (NS) acting on states with momentum in $\mathbb{Z}^{r}$. The product is always of fields of the same, R or NS, type, agreeing with the fact that it has an expansion in integral powers of $z$. Equivalently we can write the generators in terms of

$$
E^{i j}(z)=-E^{j i}(z)=\Psi^{i}(z) \Psi^{j}(z), \quad 1 \leqq i \neq j \leqq 2 r .
$$


If we consider so $(2 r+2)$, instead of so $(2 r)$, we see we can obtain the so $(2 r+1)$ subalgebra by using $\Psi^{j}(z), 1 \leqq i \leqq 2 r+1$. So we need to add to our construction of so $(2 r)$, one real fermi field $\Psi(z)=\Psi^{2 r+1}(z)$, and to work in an enlarged space generated by the $\alpha_{n}^{i}, n \in \mathbb{Z} ; e^{i \lambda \cdot q}, \lambda \in \Lambda$; and the one fermi field. More precisely, if $\mathscr{H}_{0}$ is the subspace of $\mathscr{H}$ with momentum in $\mathbb{Z}^{r}$ and $\mathscr{H}_{1}$ the subspace with momentum in $M$, we work in a space

$$
\mathscr{H}^{\prime}=\left(\mathscr{H}_{0} \otimes \mathscr{H}_{\mathrm{R}}\right) \oplus\left(\mathscr{H}_{1} \otimes \mathscr{H}_{\mathrm{NS}}\right)
$$

where $\mathscr{H}_{\mathrm{R}}$ is the space generated by a single Ramond field

$$
\Psi(z)=\sum_{n \in \mathbb{Z}} d_{n} z^{-n}, \quad \text { in } \mathscr{H}_{\mathrm{R}},
$$

and $\mathscr{H}_{\mathrm{NS}}$ by a single Neveu-Schwarz field

$$
\Psi(z)=\sum_{s \in \mathbb{Z}+1 / 2} b_{s} z^{-s} \text { in } \mathscr{H}_{\mathrm{NS}} .
$$

The field $\Psi$ is real in that

$$
b_{s}^{\dagger}=b_{-s} \quad \text { and } \quad d_{m}^{\dagger}=d_{-m} .
$$

Then we can define a representation of sô $(2 r+1)$ by adjoining to $E_{m}^{\alpha}, \alpha \in \Psi_{L}$; and $H_{m}^{i}, 1 \leqq i \leqq r$,

$$
E^{e}(z)=\Psi^{e}(z) \Psi(z)=\sum_{n \in \mathbb{Z}} E_{n}^{e} z^{-n}
$$

$e \in \Phi_{S}$. Then

$$
\begin{aligned}
{\left[H_{m}^{i}, E_{n}^{e}\right] } & =e^{i} E_{m+n}^{e}, & & \\
{\left[E_{n}^{\alpha}, E_{n}^{e}\right] } & =\varepsilon(\alpha, e) E_{m+n}^{\alpha+e}, & & \alpha+e \in \Phi_{S}, \\
& =0, & & \text { otherwise, } \\
{\left[E_{m}^{e}, E_{n}^{f}\right] } & =\varepsilon(e, f) E_{m+n}^{e+f}, & & e+f \in \Phi_{L}, \\
& =e \cdot H_{m+n}+m \delta_{m,-n}, & & e=-f, \\
& =0, & & \text { otherwise. }
\end{aligned}
$$

This construction has been known for some years [8].

We have seen that the requirement that the calculation progress without too much modification of the methods of Sect. 2 implies that $\Phi_{S}$ consists of orthogonal unit vectors, so restricting us to so $(2 r+1)$. The other simple non-simply laced algebras have short roots which are not either parallel or orthogonal. So to consider $\operatorname{sp}(n)$ or $F_{4}$, we must consider the possibility that $e \cdot f= \pm \frac{1}{2}$. In this case the fermionic operators corresponding to $e \in \Phi_{S}$ will not be independent, and nor will the additional ones we introduce.

\section{General Structure of the Vertex Representation for Non-Simply-Laced Algebras}

In this section we shall discuss an algebra $g$, with root system $\Phi$, involving roots of two lengths, the long roots $\Phi_{L}$ being of squared length 2 and the short roots $\Phi_{S}$ being 
of squared length 1 , thus excluding $G_{2}$, which will be considered in Sect. 8. Consider the consequences of the conditions (2.1) for the root system $\Phi$.

(i) If $\alpha, \beta \in \Phi_{L}$, then $\alpha \cdot \beta \in \mathbb{Z}$ and $|\alpha \cdot \beta| \leqq 2$. If $\alpha \cdot \beta \geqq 0$ then $\alpha+\beta \notin \Phi$, whereas if $\alpha \cdot \beta=-1, \alpha+\beta=\sigma_{\alpha}(\beta) \in \Phi_{L}$ and if $\alpha \cdot \beta=-2, \alpha=-\beta$. This shows that $\Phi_{L}$ is the root of a simply-laced algebra $g_{L} \subset g$ of the same rank.

(ii) If $\alpha \in \Phi_{L}$ and $e \in \Phi_{S}$, then $\alpha \cdot e \in \mathbb{Z}$ and $|\alpha \cdot e| \leqq 1$. If $\alpha \cdot e \geqq 0$ then $\alpha+e \notin \Phi$, whereas if $\alpha \cdot e=-1, \alpha+e=\sigma_{\alpha}(e) \in \Phi_{S}$. This shows that the step operators corresponding to roots $e \in \Phi_{S}$ form a representation of $g_{L}$.

(iii) If $e, f \in \Phi_{S}$, then $2 e \cdot f \in \mathbb{Z}$ and $|e \cdot f| \leqq 1$. If $e \cdot f>0$ then $e+f \notin \Phi$; if $e \cdot f=0$ either $e+f \in \Phi_{L}$ or $e+f \notin \Phi$; if $e \cdot f=-\frac{1}{2}, e+f=\sigma_{e}(f) \in \Phi_{S}$; if $e \cdot f=-1$ then $e=-f$.

The algebra $\hat{g}$ has a basis consisting of $H_{m}^{j}, E_{m}^{\alpha}, E_{m}^{e}, 1 \leqq j \leqq r, \alpha \in \Phi_{L}, e \in \Phi_{S}$, $m \in \mathbb{Z}$ and, for a level one representation, the algebra is given by Eqs. (2.24) for $H_{m}^{j}$, $E_{m}^{\alpha}, \alpha \in \Phi_{L}$, together with, for $\alpha \in \Phi_{L}, e, f \in \Phi_{S}$,

$$
\begin{aligned}
{\left[H_{m}^{i}, E_{n}^{e}\right] } & =e^{i} E_{m+n}^{e}, & & \\
{\left[E_{n}^{\alpha}, E_{n}^{e}\right] } & =\varepsilon(\alpha, e) E_{m+n}^{\alpha+e}, & & \alpha+e \in \Phi_{S}, \\
& =0, & & \text { otherwise. } \\
{\left[E_{m}^{e}, E_{n}^{f}\right] } & =\varepsilon(e, f) E_{m+n}^{e+f}, & & e+f \in \Phi_{L}, \\
& =\frac{1}{\sqrt{2}} \varepsilon(e, f) E_{m+n}^{e+f}, & & e+f \in \Phi_{S}, \\
& =e \cdot H_{m+n}+m \delta_{m+n, 0}, & & \text { if } \quad e=-f, \\
& =0, & & \text { otherwise. }
\end{aligned}
$$

In these commutation relations each $\varepsilon(\lambda, \mu), \lambda, \mu \in \Phi$, is \pm 1 . The factor $1 / \sqrt{2}$ is due to the fact that we are using a Cartan-Weyl basis rather than a Chevalley basis. In the latter basis $E^{\alpha}$ would be replaced by $F^{\alpha}=\left(2 / \alpha^{2}\right)^{1 / 2} E^{\alpha}$ and the structure constants would all be integral.

For the simply-laced algebra $\hat{g}_{L}$ we can use the usual vertex operator representation of Sect. 2,

$$
E^{\alpha}(z)=U(\alpha, z) c_{\alpha}=\sum E_{n}^{\alpha} z^{-n}
$$

and

$$
H^{i}(z)=\sum \alpha_{n}^{i} z^{-n}=\sum H_{n}^{i} z^{-n} .
$$

Following Sect. 3, we assume that the $E^{e}(z), e \in \Phi_{S}$, are given by an expression of the form

$$
E^{e}(z)=U(e, z) c_{e} \Psi_{\Omega}(z)
$$

where $c_{e}$ is a function of momentum and $\Psi_{\Omega}(z)$ is a real fermion field commuting with $\alpha_{n}^{j}$ and $e^{i \lambda \cdot q}$. It is clear from Sect. 3 that we do not need a different fermion field for each $e \in \Phi_{S}$; there we only needed one real fermion altogether.

The fermionic nature of $\Psi_{\Omega}(z)$ is recognised from the property

$$
\Psi_{\Omega}(z) \Psi_{\Omega}(\zeta)=\circ \Psi_{\Omega}(z) \Psi_{\Omega}(\zeta)_{\circ}^{\circ}-\Delta(z, \zeta), \quad|z|>|\zeta|
$$


where $\Delta(z, \zeta)=\sqrt{z \zeta} /(z-\zeta)$ or $(z+\zeta) / 2(z-\zeta)$, according as $\Psi_{\Omega}$ is of NS or R type. Thus the Laurent coefficients of $\Psi_{\Omega}$ satisfy (3.15) or (3.16) as appropriate, with the reality property (3.17). Equation (4.3c) can then be verified in the same way as Eq. $(3.25 \mathrm{~b})$, provided that

$$
\hat{c}_{e} \hat{c}_{-e}=\hat{c}_{-e} \hat{c}_{e}=1 \text {. }
$$

Considering the calculation of $\left[E_{m}^{\alpha}, E_{n}^{e}\right]$, we see that (4.6) will produce Eqs. (4.2) provided that

$$
\hat{c}_{\alpha} \hat{c}_{\gamma}=\varepsilon(\alpha, \gamma) \hat{c}_{\alpha+\gamma}=(-1)^{\alpha \cdot \gamma} \hat{c}_{\gamma} \hat{c}_{\alpha}
$$

$\alpha \in \Lambda_{R}\left(g_{L}\right), \gamma \in \Lambda_{R}(g)$, so that $\alpha \cdot \gamma \in \mathbb{Z}$, and we use the same fermion field $\Psi_{\Omega}$ for $e$ and $\sigma_{\alpha}(e)=\alpha+e$ if $\alpha \cdot e=-\frac{1}{2}$. This implies that we should use the same fermion field for short roots $e \in \Phi_{S}$ related by the Weyl reflections $\sigma_{\alpha}, \alpha \in \Phi_{L}$. These Weyl reflections generate $W\left(g_{L}\right)$, the Weyl group of $g_{L}$.

We use the Weyl group $W\left(g_{L}\right)$ to divide $\Phi_{S}$ into disjoint orbits $\Omega$ and we introduce distinct fermion fields $\Psi_{\Omega}(z)$ for each orbit. These fields will not all be independent, in the sense of anticommutating with each other for distinct $\Omega$. If $g$ is simple these orbits are all isomorphic, that is they contain the same number of roots and different orbits are related by othogonal transformations. To see this note that, because

$$
\sigma \sigma_{\alpha} \sigma^{-1}=\sigma_{\sigma(\alpha)}
$$

for any $\alpha \in \Phi$ and orthogonal transformation $\sigma$, and so in particular for $\alpha \in \Phi_{L}$ and $\sigma \in W(g)$,

$$
\sigma W\left(g_{L}\right) \sigma^{-1}=W\left(g_{L}\right) \quad \text { for } \quad \sigma \in W(g),
$$

i.e. $W\left(g_{L}\right)$ is a normal subgroup of $W(g)$. If $g$ is simple, any two $e, f \in \Phi_{S}$ can be related by a $\sigma \in W(g)$,

$$
f=\sigma(e) \text { for some } \sigma \in W(g) .
$$

From (4.11) it follows that, if $e, f$ belong to orbits $\Omega_{e}, \Omega_{f}$, of $\Phi_{S}$ under $W\left(g_{L}\right)$, respectively,

$$
\Omega_{f}=\sigma\left(\Omega_{e}\right)
$$

The elements of a given orbit $\Omega$ of $\Phi_{S}$ under $W\left(g_{L}\right)$ differ by vectors in $\Lambda_{R}\left(g_{L}\right)$. The converse is also true provided that $\Phi_{L}$ spans the root space, which is the case in particular if $g$ is simple: if $e, f \in \Phi_{S}$ differ by an element of $\Lambda_{R}\left(g_{L}\right)$ then they are in the same orbit of $\Phi_{S}$ under $W\left(g_{L}\right)$. The proof of this is a little more involved. If $e-f \in \Lambda_{R}\left(g_{L}\right),(e-f)^{2} \in 2 \mathbb{Z}$. So $e \cdot f= \pm 1,0$, i.e. either $e= \pm f$ or $e \cdot f=0$. If $e \cdot f=0, \alpha=e-f$ is a point of $\Lambda_{R}\left(g_{L}\right)$ of squared length 2 and so is a long root. But then $\sigma_{\alpha}(e)=f$. Thus it remains to show that $-e$ is on the same orbit as $e$. If $\Phi_{L}$ spans the root space, we can find $\beta \in \Phi_{L}$ with $\beta \cdot e \neq 0$. Then $\beta \cdot e= \pm 1$ and we may reverse the sign of $\beta$ if necessary to obtain $\beta \cdot e=+1$. We now construct short roots in the same orbit as $e$ by a sequence of reflections, ending up with $-e$. Firstly,

$$
\delta=\sigma_{\beta}(e)=e-\beta \in \Omega_{e},
$$

has $\delta \cdot \beta=-1$ and $\delta \cdot e=0$. Then

$$
\sigma_{\delta}(\beta)=\beta-2(\delta \cdot \beta) \delta=\beta+2 \delta=e+\delta \in \Phi_{L},
$$


and if we reflect $\delta$ using the corresponding $\sigma_{e+\delta} \in W\left(g_{L}\right)$,

$$
\sigma_{e+\delta}(\delta)=\delta-(e+\delta) \cdot \delta(e+\delta)=-e \in \Omega_{e},
$$

establishing the desired result.

So, at least when $g$ is simple, we can identify an orbit $\Omega$ of $\Phi_{S}$ under $W\left(g_{L}\right)$ with the points of unit length in a coset $\Lambda_{a}$ of $\Lambda_{R}(g) / \Lambda_{R}\left(g_{L}\right)$. Not all such cosets will have points of unit length, in particular $\Lambda_{0}=\Lambda_{R}(g)$ has none. To exemplify this, let us enumerate the various cases for $g$ simple. These are so $(2 r+1), r \geqq 2 ; \operatorname{sp}(r), r \geqq 2$; and $F_{4}$. For $g=\operatorname{so}(2 r+1)$.

$$
\begin{aligned}
\Lambda_{R}(g) & =\mathbb{Z}^{r} \\
\Lambda_{0} & =\left\{\left(n_{1}, \ldots, n_{r}\right): n_{i} \in \mathbb{Z}, \sum n_{i} \in 2 \mathbb{Z}\right\} .
\end{aligned}
$$

Thus there are two cosets $\Lambda_{0}$ and $\Lambda_{1}$, only the latter having points of unit length. This corresponds to the single real fermi field we introduced in Sect. 3. For $g=\operatorname{sp}(r)$,

$$
\begin{aligned}
\Lambda_{R}(g) & =\left\{2^{-1 / 2}\left(n_{1}, \ldots, n_{r}\right): n_{i} \in \mathbb{Z}, \sum n_{i} \in 2 \mathbb{Z}\right\}, \\
\Lambda_{0} & =\left\{2^{-1 / 2}\left(n_{1}, \ldots, n_{r}\right): n_{i} \in 2 \mathbb{Z}\right\} .
\end{aligned}
$$

Thus there are $2^{r-1}$ cosets of $\Lambda_{0}$ in $\Lambda_{R}(\operatorname{sp}(r))$ and $\frac{1}{2} r(r-1)$ of them have points of unit length. For $g=F_{4}$.

$$
\begin{aligned}
\Lambda_{R}(g)= & \left\{\left(n_{1}, n_{2}, n_{3}, n_{4}\right): \quad \text { either } \quad n_{i} \in \mathbb{Z}, 1 \leqq i \leqq 4,\right. \\
& \text { or } \left.\quad n_{i} \in \mathbb{Z}+\frac{1}{2}, 1 \leqq i \leqq 4\right\} \\
\Lambda_{0}= & \left\{\left(n_{1}, n_{2}, n_{3}, n_{4}\right): n_{i} \in \mathbb{Z}, \sum n_{i} \in 2 \mathbb{Z}\right\} .
\end{aligned}
$$

In this case, there are 4 cosets of $\Lambda_{0}$ in $\Lambda_{R}$ and 3 of them, all except $\Lambda_{0}$, have points of unit length.

Note that it follows from the facts that if $f \in \Omega$ then $-f \in \Omega$ and that if $e$, $f \in \Omega, e \neq \pm f$ then $e \cdot f=0$ and $e \pm f \in \Lambda_{R}\left(g_{L}\right)$, that an orbit $\Omega$ consists of points $\pm f_{i}$, $1 \leqq i \leqq \operatorname{dim} \Omega$, where $f_{i} \cdot f_{j}=\delta_{i j}$, and with $\pm f_{i} \pm f_{j} \in \Lambda_{R}\left(g_{L}\right)$ if $i \neq j$. If $\xi_{i}, 1 \leqq i \leqq r$, is an orthogonal basis for $r$-dimensional space, the single orbit for so $(2 r+1)$ consists of the $2 r$ vectors $\left\{ \pm \xi_{i}: 1 \leqq i \leqq r\right\}$; the $\frac{1}{2} r(r-1)$ orbits for $\mathrm{sp}(r)$ can be labelled by integers $i, j, 1 \leqq i<j \leqq r$ and consist of the 4 vectors $\left( \pm \xi_{1} \pm \xi_{j}\right) / \sqrt{2}$; the 3 orbits for $F_{4}$ each consist of 8 vectors and are

$$
\begin{aligned}
& \left\{ \pm \xi_{i}: 1 \leqq i \leqq 4\right\}, \\
& \left\{ \pm \frac{1}{2} \xi_{1} \pm \frac{1}{2} \xi_{2}+\frac{1}{2} \xi_{3} \pm \frac{1}{2} \xi_{4}: \text { even number of }+ \text { signs }\right\} \\
& \left\{ \pm \frac{1}{2} \xi_{1} \pm \frac{1}{2} \xi_{2}+\frac{1}{2} \xi_{3} \pm \frac{1}{2} \xi_{4}: \text { odd number of }+ \text { signs }\right\}
\end{aligned}
$$

We see that in each case, the orbits can be labelled by $\Omega_{i j} \equiv \Omega_{j i}, 1 \leqq i<j \leqq n+1$, where $n=1$ for so $(2 r+1), n=r-1$ for $\operatorname{sp}(r)$ and $n=2$ for $F_{4}$, i.e. the number of short simple roots. Since $2 \gamma \in \Lambda_{R}\left(g_{L}\right)$ if $\gamma \in \Lambda_{R}(g)$, we have that

$$
\Lambda_{R}(g) / \Lambda_{R}\left(g_{L}\right)=\left(\mathbb{Z}_{2}\right)^{n},
$$

and the orbits $\Omega_{i j}$ correspond to the $\frac{1}{2} n(n+1)$ of these cosets with points of unit length. Further, if $e \in \Omega_{i j}$ and $f \in \Omega_{k l}$ and $\Omega_{i j} \neq \Omega_{k l}$, either $\{i, j\}$ and $\{k, l\}$ have no 
common value, in which case

$$
e \cdot f=0 \quad \text { and } \quad \pm e \pm f \notin \Lambda_{R}\left(g_{L}\right),
$$

or they have one common value, $i=k$, say, in which case

$$
|e \cdot f|=\frac{1}{2} \text { and } e \mp f \in \Omega_{j l},
$$

the sign depending on whether $e \cdot f= \pm \frac{1}{2}$.

Having analysed the structure of these orbits $\Omega$ we are in a position to consider what properties the fermi fields $\Psi_{\Omega}(z)$ should have in order that Eqs. (4.3) will hold. Take $e, f \in \Phi_{S}$ in orbits $\Omega_{1}, \Omega_{2}$, respectively. If $e \cdot f \geqq 0, E^{e}$ and $E^{f}$ should commute. Which of the other possibilities obtains depends on the relationship of the orbits $\Omega_{1}, \Omega_{2}$. Since $\alpha \cdot e \in \mathbb{Z}$ if $\alpha \in \Lambda_{0}$, whether $e \cdot f \in \mathbb{Z}$ or $\mathbb{Z}+\frac{1}{2}$ depends only on the relation of $\Omega_{1}$ to $\Omega_{2}$; in the former case we shall write $\Omega_{1} \cdot \Omega_{2} \in \mathbb{Z}$ and in the latter case $\Omega_{1} \cdot \Omega_{2} \in \mathbb{Z}+\frac{1}{2}$. In this latter case $|e \cdot f|=\frac{1}{2}$ and either $e \pm f \in \Phi_{S}$, and so in some third orbit $\Omega_{3}$, whilst in the former case $\pm e \pm f \in \Psi_{L}$ if and only if $\Omega_{1}=\Omega_{2}$. Because the coset of $\Lambda_{R}(g) / \Lambda_{0}$ which $e \pm f$ is in depends only on the cosests of $\pm e, \pm f$, and so on $\Omega_{1}, \Omega_{2}$, the coset $\Omega_{3}$ depends only on the $\Omega_{1}, \Omega_{2}$ with $\Omega_{1} \cdot \Omega_{2} \in \mathbb{Z}+\frac{1}{2}$, and not on the points $e, f$ chosen in them, and is given by the coset multiplication rule (4.17).

Looking at Eq. (3.1), we see that we need the following form for the operator product of two fermi fields in order to stand any chance of getting the desired commutation relations:

$$
\begin{aligned}
& \Psi_{\Omega_{1}}(z) \Psi_{\Omega_{2}}(\zeta)=\Sigma\left(\Omega_{1}, \Omega_{2}\right) \Psi_{\Omega_{2}}(\zeta) \Psi_{\Omega_{1}}(z), \\
& \text { regular at } z=\zeta, \quad \text { if } \Omega_{1} \cdot \Omega_{2} \in \mathbb{Z}, \Omega_{1} \neq \Omega_{2},
\end{aligned}
$$

where $\Sigma$ takes the values \pm 1 ;

$$
\Psi_{\Omega}(z) \Psi_{\Omega}(\zeta)=R_{\Omega}(z, \zeta), \quad|\zeta|<|z|,
$$

where $R_{\Omega}(z, \zeta)$ is an antisymmetric function of $z, \zeta$, having a simple pole of residue $z$ at $\zeta$ [see Eq. (4.7)];

$$
\begin{aligned}
\Psi_{\Omega_{1}}(z) & \Psi_{\Omega_{2}}(\zeta)=(z-\zeta)^{-1 / 2}\left\{\eta_{+}\left(\Omega_{1}, \Omega_{2}\right) R_{\Omega_{1}, \Omega_{2}}^{+}(z, \zeta)\right. \\
& \left.+(z-\zeta) \eta_{-}\left(\Omega_{1}, \Omega_{2}\right) R_{\Omega_{1}, \Omega_{2}}^{-}(z, \zeta)\right\}, \quad|\zeta|<|z|, \quad \text { if } \quad \Omega_{1} \cdot \Omega_{2} \in \mathbb{Z}+\frac{1}{2},
\end{aligned}
$$

where $R_{\Omega_{1}, \Omega_{2}}^{ \pm}(z, \zeta)$ are symmetric under the simultaneous interchange of $\Omega_{1}$ with $\Omega_{2}$ and $z$ with $\zeta$, and

$$
R_{\Omega_{1}, \Omega_{2}}(z, z)=\frac{z^{1 / 2}}{\sqrt{2}} \Psi_{\Omega_{3}}(z) .
$$

The $c$-number functions $\Sigma, \eta_{ \pm}$must have appropriate symmetry properties to ensure that we actually get commutation relations. To see what these should be, suppose that

$$
\hat{c}_{\gamma} \hat{c}_{\delta}=\varepsilon_{0}(\gamma, \delta) \hat{c}_{\gamma+\delta^{\prime}}
$$

for $\gamma, \delta \in \Lambda_{R}(g)$. Notice that, comparing with what we have said before in Eqs. (4.8) and (4.9), $\varepsilon_{0}(\gamma, \delta)$ must coincide with $\varepsilon(\gamma, \delta)$ when both gamma and delta are in $\Lambda_{0} \cup \Lambda_{a}$, where $\Lambda_{a}$ is one of the $2^{n}-1$ cosets in (4.17) distinct from $\Lambda_{0}$, but may, as 
we shall see, differ if $\gamma$ and $\delta$ are in cosets $\Lambda_{a}$ and $\Lambda_{b}$, respectively, distinct from each other and $\Lambda_{0}$. Then we need

$$
\varepsilon_{0}\left(e_{1}, e_{2}\right) \eta_{ \pm}\left(\Omega_{1}, \Omega_{2}\right)=(-1)^{e_{1} \cdot e_{2} \mp 1 / 2} \varepsilon_{0}\left(e_{2}, e_{1}\right) \eta_{ \pm}\left(\Omega_{2}, \Omega_{1}\right)
$$

if $e_{i} \in \Omega_{i}$, with $\Omega_{1} \cdot \Omega_{2} \in \mathbb{Z}+\frac{1}{2}$, and

$$
\begin{array}{lll}
\varepsilon_{0}\left(e_{1}, e_{2}\right)=(-1)^{e_{1} \cdot e_{2}} \varepsilon_{0}\left(e_{2}, e_{1}\right) \Sigma\left(\Omega_{1}, \Omega_{2}\right), & \text { if } \quad \Omega_{1} \cdot \Omega_{2}=0, \\
\varepsilon_{0}\left(e_{1}, e_{2}\right)=-(-1)^{e_{1} \cdot e_{2}} \varepsilon_{0}\left(e_{2}, e_{1}\right), & \text { if } \quad \Omega_{1}=\Omega_{2} .
\end{array}
$$

If conditions (4.17) to (4.23) hold we obtain the algebra (4.1)-(4.3) with

$$
\begin{aligned}
\varepsilon\left(\gamma_{1}, \gamma_{2}\right) & =\varepsilon_{0}\left(\gamma_{1}, \gamma_{2}\right), & & \text { if either } \gamma_{1} \text { or } \gamma_{2} \in \Phi_{L}, \\
& =\varepsilon_{0}\left(\gamma_{1}, \gamma_{2}\right) \eta_{+}\left(\Omega_{1}, \Omega_{2}\right), & & \text { if } \gamma_{1} \cdot \gamma_{2}=-\frac{1}{2},
\end{aligned}
$$

where $c_{i} \in \Omega_{i}$, and $\varepsilon(\gamma, \gamma)=1$. To complete the construction we need to find fermi fields $\Psi_{\Omega}(z)$ with the correct properties, and suitable cocycle functions $\varepsilon_{0}, \eta_{+}, \eta_{-}$, and $\Sigma$. The former depends on the latter, so we discuss this first in Sect. 5, constructing the fermi fields in Sect. 6.

\section{Construction of Cocycles}

In order to construct the cocycles needed for Sect. 4 we have to consider the theory of such objects in some detail; much of what we say is based on ref. [1]. We consider operators $\hat{c}_{x}$, defined on momentum states $|y\rangle$ for $y \in \Lambda$, a certain lattice, by

$$
\hat{c}_{x}|y\rangle=\varepsilon(x, y)|x+y\rangle,
$$

where $\varepsilon(x, y) \in \mathbb{C}$. Such an $\varepsilon$ will satisfy

$$
\hat{c}_{x} \hat{c}_{y}=\varepsilon(x, y) \hat{c}_{x+y},
$$

provided that

$$
\varepsilon(x, y+z) \varepsilon(y, z)=\varepsilon(x, y) \varepsilon(x+y, z) .
$$

Usually $\varepsilon(x, y)= \pm 1$, but we shall need to consider the more general case where $\varepsilon(x, y)) \in Z$, some finite subgroup of the group of complex numbers of unit modulus. Given any set of operators $\hat{c}_{x}, x \in \Lambda$, satisfying (5.2) we obtain a function $\varepsilon$ satisfying (5.3) and, conversely, given an $\varepsilon$ satisfying (5.3), we can use (5.1) to define operators satisfying (5.2).

The purpose of introducing the $\hat{c}_{x}$ and $\varepsilon(x, y)$ is to obtain a certain specified symmetry factor $S(x, y)$ by the interchange of certain operators, defined by

$$
\varepsilon(x, y)=S(x, y) \varepsilon(y, x),
$$

or

$$
\hat{c}_{x} \hat{c}_{y}=S(x, y) \hat{c}_{y} \hat{c}_{x} .
$$

For example, in the case of the simply-laced algebras of Sect. $2, \Lambda=\Lambda_{R}(g)$ and $S(x, y)=(-1)^{x \cdot y}$, whilst in the case of the cubic lattice $\Lambda=\mathbb{Z}^{r}$ in Sect. 3, 
$S(x, y)=(-1)^{x \cdot y+x^{2} y^{2}}$. Again, we shall be looking at more general cases where $S(x, y) \in Z \subset \mathbb{C}$. The symmetry factor, as a result of (5.4), also satisfies (5.3),

$$
S(x, y+z) S(y, z)=S(x, y) S(x+y, z) .
$$

However, by commuting $\hat{c}_{z}$ through (5.2), we see that it has the more restrictive additivity property

$$
S(x+y, z)=S(x, z) S(y, z),
$$

which implies (5.6). Further, (5.5) implies

and

$$
\begin{array}{r}
S(x, y) S(y, x)=1, \\
S(x, x)=1 .
\end{array}
$$

Properties (5.7) are the definitive ones for a symmetry factor. If $e_{i}, 1 \leqq i \leqq r$, is a basis for $\Lambda$, properties (5.7) mean that $S(x, y)$ is determined in terms of

$$
S_{i j}=S\left(e_{i}, e_{j}\right)
$$

where

$$
S_{i j}=S_{j i}^{-1} \quad \text { and } \quad S_{i i}=1
$$

by

$$
S(x, y)=\prod_{i . j} S_{i j}^{x_{i} \cdot y_{j}}
$$

where $x=\sum x_{i} e_{i}$ and $y=\sum y_{j} e_{j}$. Conversely given $S_{i j} \in Z$, satisfying (5.9), Eq. (5.10) defines a symmetry factor satisfying conditions (5.7).

Given an $\varepsilon(x, y)$ satisfying (5.3) and (5.4) for a given symmetry factor $S(x, y)$, we can obtain another one satisfying these equations with the same symmetry factor by the "gauge transformation"

$$
\varepsilon^{\prime}(x, y)=\frac{u(x) u(y)}{u(x+y)} \varepsilon(x, y),
$$

where $u(x) \in Z$ for $x \in \Lambda$. This transformation corresponds to changing from $\hat{c}_{x}$ to

$$
\hat{c}_{x^{\prime}}=u(x) \hat{c}_{x}
$$

A central result is that $S(x, y)$ determines $\varepsilon(x, y)$ up to gauge transformations of this type, and, for any $S(x, y)$ satisfying conditions (5.7) [or equivalently any set of $S_{i j}$ satisfying (5.9)], we can construct an $\varepsilon(x, y)$ satisfying (5.3) and (5.4).

To prove the existence of an $\varepsilon(x, y)$ for given $S_{i j}$, we introduce operators $\hat{\gamma}_{i}$ satisfying

$$
\hat{\gamma}_{i} \hat{\gamma}_{j}=S_{i j} \hat{\gamma}_{j} \hat{\gamma}_{i}
$$

This can be done with matrices of finite dimension. We can then introduce

$$
\hat{\gamma}_{x}=\hat{\gamma}_{1}^{x_{1}} \hat{\gamma}_{2}^{x_{2}} \ldots \hat{\gamma}_{r}^{x_{r}}
$$

It follows from (5.13) that

$$
\hat{\gamma}_{x} \hat{\gamma}_{y}=\varepsilon_{0}(x, y) \hat{\gamma}_{x+y}
$$


with $\varepsilon_{0}(x, y) \in Z$, and then $\varepsilon_{0}(x, y)$ automatically satisfies (5.3), and (5.4) with $S(x, y)$ satisfying $(5.8)$, which determines it uniquely. To show that this $\varepsilon_{0}(x, y)$ is unique up to gauge transformation, suppose $\varepsilon(x, y)$ satisfies (5.3) and (5.4) and define $\hat{c}_{x}$ by (5.1). Then $\hat{\gamma}_{i}=\hat{c}_{e_{i}}$ satisfies (5.13) and the operators $\hat{\gamma}_{x}$, defined by (5.14), must satisfy

$$
\hat{c}_{x}=u(x) \hat{\gamma}_{x},
$$

for some $u(x) \in Z$. Then it follows that

as required.

$$
\varepsilon(x, y)=\frac{u(x) u(y)}{u(x+y)} \varepsilon_{0}(x, y),
$$

We need to exploit the ability to make gauge transformations to ensure that

$$
\hat{c}_{x}^{\dagger}=\hat{c}_{-x}
$$

and

$$
\hat{c}_{0}=1 \text {. }
$$

To do this note that if we put $y=0$ in (5.3) showing that

$$
\varepsilon(0, z)=\varepsilon(x, 0)
$$

all have some common value, which can be set to 1 by a choice of $u(0)$. This ensures (5.19). Since $\hat{c}_{x}$ is clearly unitary, provided that $|\varepsilon(x, y)|=1$, because it maps one orthonormal unit basis into another, (5.18) will follow from

$$
\hat{c}_{x} \hat{c}_{-x}=1 \text {. }
$$

This condition is equivalent to

$$
\varepsilon(x,-x)=1,
$$

which can be arranged by a gauge transformation by sultably choosing $u(x) u(-x)$, fixing half the $u(x)$ relative to the rest.

Having arranged, by gauge choice, that (5.20) and (5.22) hold, we are still free to make further gauge transformations $u(x)$ subject to

$$
u(x) u(-x)=u(0)=1 .
$$

However, it is not usually necessary to specify the gauge choice further.

Now let us consider the application of these results to the construction of a suitable cocycle $\varepsilon_{0}$, as in Eq. (4.23), for the lattice $\Lambda_{R}(g)$ of Sect. 4. From what we have said, $\varepsilon_{0}$ is determined by specifying its symmetry factor $S_{0}$. From Eqs. (4.8), (4.9), and (4.26), we see that we need

$$
S_{0}(x, y)=(-1)^{x \cdot y+x^{2} y^{2}}
$$

if $x, y \in \Lambda_{0} \cup \Lambda_{a}$, where $\Lambda_{0}=\Lambda_{R}\left(g_{L}\right)$ and $\Lambda_{a}$ is a coset of $\Lambda_{R}(g)$ with respect to $\Lambda_{0}$. It is not so clear what happens if $x, y \in \Lambda_{R}\left(g_{L}\right)$ with $x \cdot y \in \mathbb{Z}+\frac{1}{2}$. In fact we must choose $S_{0}(x, y)= \pm i$ in such cases because, since $\pm e$ are in the same orbit $\Omega$ if $e \in \Phi_{S}, 2 e \in \Lambda_{R}\left(g_{L}\right)$ for each e $\in \Phi_{S}$, and hence $2 \Lambda_{R}(g) \subset \Lambda_{R}\left(g_{L}\right)$. This implies that if $x \in \Lambda_{R}(g), 2 x \in \Lambda_{R}\left(g_{L}\right)$ so that

$$
S_{0}(x, y)^{2}=S_{0}(2 x, y)=(-1)^{2 x \cdot y} .
$$


If $x \cdot y \in \mathbb{Z}+\frac{1}{2}$ this equals -1 , showing that

$$
S_{0}(x, y)= \pm i \quad \text { if } \quad x \cdot y \in \mathbb{Z}+\frac{1}{2} .
$$

It is straightforward to construct an $S_{0}(x, y)$ with these properties. Take a basis $\alpha_{1}, \ldots, \alpha_{r}$ of simple roots for $g$; this provides a basis for $\Lambda_{R}(g)$. It is straightforward to check, for $g=\operatorname{so}(2 r+1), \operatorname{sp}(r)$ and $F_{4}$, that a basis for $\Lambda_{R}\left(g_{L}\right)$ is provided by $\beta_{1}, \ldots, \beta_{r}$, where $\beta_{j}=\alpha_{j}$ if $\alpha_{j} \in \Phi_{L}$ and $\beta_{j}=2 \alpha_{j}$ if $\alpha_{j} \in \Phi_{S}$. [More generally, if $\Lambda / \Lambda_{0}=\left(\mathbb{Z}_{2}\right)^{n}$ and $\left\{\alpha_{j}\right\}$ is a basis for $\Lambda$, the number of basis elements $\alpha_{i} \in \Lambda_{0}$ is $n$ and $\left\{\beta_{j}\right\}$, defined by $\beta_{j}=\alpha_{j}$ unless $\alpha_{j} \in \Lambda_{0}$, in which case $\beta_{j}=2 \alpha_{j}$, generates $\Lambda_{0}$, since it generates a sublattice of $\Lambda_{0}$ whose fundamental domain has the same volume as $\Lambda_{0}$.] We define $S_{0}(x, y)$ by specifying that

$$
S_{0}\left(\alpha_{i}, \alpha_{j}\right)=(-1)^{\alpha_{i} \cdot \alpha_{j}} \quad \text { if } \quad \alpha_{i} \cdot \alpha_{j} \in \mathbb{Z}, \quad i \neq j,
$$

and

$$
S_{0}\left(\alpha_{i}, \alpha_{j}\right)=-S_{0}\left(\alpha_{j}, \alpha_{i}\right)= \pm i \quad \text { if } \quad \alpha_{i} \cdot \alpha_{j}=-\frac{1}{2}
$$

and we are free to choose either sign in $(5.27 \mathrm{~b})$ for each pair $i<j$. For example, a particular choice would be

$$
S_{0}\left(\alpha_{i}, \alpha_{j}\right)=e^{i \pi \alpha_{i} \cdot \alpha_{j}}, \quad i<j .
$$

It follows from (5.28) that

$$
S_{0}\left(\alpha_{i}, \beta_{j}\right)=S_{0}\left(\beta_{j}, \alpha_{i}\right)=(-1)^{\alpha_{i} \cdot \beta_{j}},
$$

and this implies (5.24).

Now we consider whether the conditions (4.24) and (4.25) on the functions $\Sigma\left(\Omega_{1}, \Omega_{2}\right), \eta_{ \pm}\left(\Omega_{1}, \Omega_{2}\right)$ of Eqs. (4.19) and (4.21) are self-consistent. If we introduce symmetry functions $\Sigma_{ \pm}$for $\eta_{ \pm}$by

$$
\eta_{ \pm}\left(\Omega_{1}, \Omega_{2}\right)=\Sigma_{ \pm}\left(\Omega_{1}, \Omega_{2}\right) \eta_{ \pm}\left(\Omega_{2}, \Omega_{1}\right)
$$

the conditions (4.24) to (4.26) are equivalent to

$$
\begin{array}{lll}
S_{0}\left(e_{1}, e_{2}\right) \Sigma_{ \pm}\left(\Omega_{1}, \Omega_{2}\right)=(-1)^{e_{1} \cdot e_{2} \mp 1 / 2}, & \text { if } & \Omega_{1} \cdot \Omega_{2} \in \mathbb{Z}+\frac{1}{2}, \\
S_{0}\left(e_{1}, e_{2}\right) \Sigma\left(\Omega_{1}, \Omega_{2}\right)=1, & \text { if } \quad e_{1} \cdot e_{2}=0,
\end{array}
$$

and

$$
S_{0}\left(e_{1}, e_{2}\right)=-(-1)^{e_{1} \cdot e_{2}} \quad \text { if } \quad e_{1}, e_{2} \in \Omega .
$$

To show that these conditions are consistent we need to show that, for given $\Omega_{1}, \Omega_{2}$, $S_{0}\left(e_{1}, e_{2}\right)(-1)^{-e_{1} \cdot e_{2}}$ is independent of the choices of $e_{i} \in \Omega_{i}$. This follows from Eqs. (5.24), (5.7a) and the fact that elements of $\Omega$ differ by vectors in $\Lambda_{R}\left(g_{L}\right)$. Thus, if $f_{i}=e_{i}+\beta_{i}$,

$$
S_{0}\left(f_{1}, f_{2}\right)=S_{0}\left(e_{1}, e_{2}\right)(-1)^{e_{1} \cdot \beta_{2}+e_{2} \cdot \beta_{1}+\beta_{1} \cdot \beta_{2}}=S_{0}\left(e_{1}, e_{2}\right)(-1)^{f_{1} \cdot f_{2}-e_{1} \cdot e_{2}},
$$

establishing the desired result. [Condition (5.31c) hence holds because $S_{0}\left(e_{1}, e_{1}\right)=1$.] We will construct $\eta_{ \pm}$with these properties in Sect. 6 .

Finally, note that, if we have constructed functions $\hat{c}_{x}$, associated with the points $x \in \Lambda$, acting on momentum states $|y\rangle$ for $y \in \Lambda$, we can extend $\hat{c}_{x}$ so that it 
acts on states with momentum in some larger lattice $\Lambda^{\prime} \supset \Lambda$, by dividing $\Lambda^{\prime}$ into cosets $\Lambda_{j}^{\prime}$ with respect to $\Lambda$ and, choosing a vector $\lambda_{j} \in \Lambda_{j}^{\prime}$, then replacing $\hat{c}_{x}$ by

$$
\sum_{j} e^{i q \cdot \lambda_{J}} \hat{c}_{x} e^{-i q \cdot \lambda_{j}}
$$

It is straightforward to check that this generalised $\hat{c}_{x}$ has the property (5.2).

\section{Construction of the Fermions}

In Sect. 4, we saw that to construct the level 1 representation for a simple algebra $g$, we need to introduce a fermion field $\Psi_{\Omega}(z)$ for each orbit $\Omega$ of $\Phi_{S}$ under the Weyl group of $g_{L}$, and that these orbits could be labelled by $\Omega_{i j}, 1 \leqq i<j \leqq n+1$, where $n=1$ for so $(2 r+1), r-1$, for $\operatorname{sp}(r)$, and 2 for $F_{4}$, i.e. the number of short roots of $g$. To do this we use fermions associated with the points of unit length on

$$
\Lambda_{n}=\frac{1}{\sqrt{2}} \Lambda_{R}(\operatorname{su}(n+1))
$$

the root lattice of $\mathrm{su}(n+1)$ rescaled by a factor of $1 / \sqrt{2}$.

Taking an orthonormal basis $\left.\xi_{i}, 1 \leqq i \leqq i \leqq n+1\right)$, for $\mathbb{R}^{n+1}$, we may take $\Lambda_{n}$ to consist of the points

$$
\frac{1}{\sqrt{2}}\left(m_{1} \xi_{1}+m_{2} \xi_{2}+\ldots+m_{n+1} \xi_{n+1}\right), \quad \sum m_{j}=0 .
$$

A basis for $\Lambda_{n}$ is provided by

$$
\alpha_{i}^{\prime}=\frac{1}{\sqrt{2}}\left(\xi_{i}-\xi_{i+1}\right), \quad 1 \leqq i \leqq n .
$$

The points of $\Phi_{S}^{\prime}$ unit length on $\Lambda_{n}$ are

$$
\gamma_{i j}=\frac{1}{\sqrt{2}}\left(\xi_{i}-\xi_{j}\right), \quad i \neq j
$$

We shall write $\gamma_{i j}>0$ if $i>j$ and $\gamma_{j i}<0$ if $i<j$. These divide into $\frac{1}{2} n(n+1)$ pairs

$$
\Omega_{i j}^{\prime}=\left\{ \pm \gamma_{i j}, \gamma_{j i}=-\gamma_{i j}: i<j\right\},
$$

which correspond to different cosets of $\Lambda_{n} / 2 \Lambda_{n}$ which, like $\Lambda_{R}(g) / \Lambda_{R}\left(g_{L}\right)$, is isomorphic to $\left(\mathbb{Z}_{2}\right)^{n}$. We associate to each orbit $\Omega$ one of these pairs $\Omega^{\prime}$, by associating $\Omega_{i j}^{\prime}$ to $\Omega_{i j}$.

To write down a fermion vertex operator for each $\gamma \in \Phi_{S}^{\prime}$ we need to define suitable functions $\hat{c}_{x}^{\prime}$ of momentum, $x \in \Lambda_{n}$.

These will be specified up to gauge equivalence by specifying a symmetry factor $S_{0}^{\prime}(x, y), x, y \in \Lambda_{n}$. We shall see that it is crucial to do this in a way correlated with the cocycle to be used for $\Lambda_{R}(g)$ or, more particularly, its symmetry factor $S_{0}$. To do 
this note that, since $n$ is the number of short simple roots of $g$, we may order the basis of simple roots $\alpha_{1}, \ldots, \alpha_{r}$ for $g$ so that it has the following properties:

$$
\alpha_{i} \in \Phi_{S}, \quad 1 \leqq i \leqq n ; \quad \alpha_{i} \in \Phi_{L}, \quad n<i \leqq r ;
$$

(ii) the lattice generated by $\alpha_{1}, \ldots, \alpha_{r}$ contains points in all the orbits $\Omega$ of $\Phi_{S}$ with respect to the Weyl group $W\left(g_{L}\right)$;

$$
\alpha_{i} \cdot \alpha_{j}=\alpha_{i}^{\prime} \cdot \alpha_{j}^{\prime}, \quad 1 \leqq i, j \leqq n,
$$

equalling $-\frac{1}{2}$ if $|i-j|=1,1$ if $i=j$ and 0 otherwise. We derine $S_{0}^{\prime}(x, y)$ by

$$
S_{0}^{\prime}\left(\alpha_{i}^{\prime}, \alpha_{j}^{\prime}\right)=S_{0}\left(\alpha_{i}, \alpha_{j}\right)
$$

Then $S_{0}^{\prime}$ has similar properties to $S_{0}$; in particular

$$
S_{0}^{\prime}(x, y)=(-1)^{x \cdot y},
$$

if either $x$ or $y \in 2 \Lambda_{n}$ and, if $e_{j}^{\prime}, f_{j}^{\prime} \in \Omega_{j}^{\prime}$,

$$
S_{0}^{\prime}\left(f_{1}^{\prime}, f_{2}^{\prime}\right)=S_{0}^{\prime}\left(e_{1}^{\prime}, e_{2}^{\prime}\right)(-1)^{f_{1}^{\prime} f_{2}^{\prime}-e_{1}^{\prime} \cdot e_{2}^{\prime}} .
$$

In consequence of this and Eq. (5.32), if $e_{j} \in \Omega_{j}$, the orbit in $\Lambda_{R}(g)$ corresponding to $\Omega_{j}^{\prime}$ in $\Lambda_{n}$,

$$
S_{0}^{\prime}\left(e_{1}^{\prime}, e_{2}^{\prime}\right)=S_{0}\left(e_{1}, e_{2}\right),
$$

provided that $e_{1} \cdot e_{2}=e_{1}^{\prime} \cdot e_{2}^{\prime}$. We suppose that we define from this $S_{0}^{\prime}, \hat{c}_{x}^{\prime}$ and $\varepsilon_{0}^{\prime}$ using the procedures of Sect. 5 .

We are now in a position to write down fermion fields $\Psi_{\gamma}(z)$ for $\gamma \in \Phi_{S}^{\prime}$, acting in a bosonic Fock space $\mathscr{K}^{\prime}$, generated by annihilation and creation operators $\alpha_{m}^{\prime j}$ from states $|x\rangle, x \in \Lambda_{n}$,

$$
\Psi_{\gamma}(z)=U^{\prime}(\gamma, z) \hat{c}_{\gamma}^{\prime}
$$

[with $U^{\prime}$ defined in terms of $\alpha_{m}^{\prime j}, e^{i \gamma \cdot q^{\prime}}, p^{\prime}$ in an analogous way to $U$ in Eq. (2.7)]. From (3.1), the product of such fields has the structure,

$$
\Psi_{\gamma_{1}}(z) \Psi_{\gamma_{2}}(z)=\varepsilon_{0}^{\prime}\left(\gamma_{1}, \gamma_{2}\right)(z-\zeta)^{\gamma_{1} \cdot \gamma_{2}} \Psi_{\gamma_{1}, \gamma_{2}}(z, \zeta), \quad|\zeta|<|z|,
$$

where $\Psi_{\gamma_{1}, \gamma_{2}}$ has the properties:

$$
\Psi_{\gamma_{1}, \gamma_{2}}(z, \zeta)=\Psi_{\gamma_{2}, \gamma_{1}}(\zeta, z)
$$

and is regular at $z=\zeta$ with

$$
\begin{gathered}
\Psi_{\gamma,-\gamma}(z, \zeta)=z+O(z-\zeta) \quad \text { at } z=\zeta, \\
\Psi_{\gamma_{1}, \gamma_{2}}(z, \zeta)=\sqrt{z} \Psi_{\gamma_{1}+\gamma_{2}}(z, \zeta)+O(z-\zeta), \quad \text { if } \quad \gamma_{1} \cdot \gamma_{2}=-\frac{1}{2} .
\end{gathered}
$$

This provides one complex field for each orbit because

$$
\Psi_{\gamma}(z)^{\dagger}=\Psi_{-\gamma}\left(1 / z^{*}\right) .
$$

To get a single real field associated with the orbits $\Omega$ of $\Phi_{\mathrm{S}}$ we set

$$
\Psi_{\Omega}(z)=\frac{1}{\sqrt{2}}\left\{\Psi_{\gamma}(z)+\Psi_{-\gamma}(z)\right\},
$$


where $\gamma \in \Omega^{\prime}$ the orbit of $\Lambda_{n}$ corresponding to $\gamma$. In considering the products of such fields, we need to consider the relationship between $\varepsilon_{0}^{\prime}\left(\gamma_{1}, \gamma_{2}\right)$ and $\varepsilon_{0}^{\prime}\left(-\gamma_{1},-\gamma_{2}\right)$. If we set

$$
\varepsilon^{\prime}\left(\gamma_{1}, \gamma_{2}\right)=\varepsilon_{0}^{\prime}\left(\gamma_{1}, \gamma_{2}\right) / \varepsilon_{0}^{\prime}\left(-\gamma_{1},-\gamma_{2}\right)
$$

the corresponding symmetry factor $S^{\prime}\left(\gamma_{1}, \gamma_{2}\right)$ is given by

$$
S^{\prime}\left(\gamma_{1}, \gamma_{2}\right)=S_{0}^{\prime}\left(\gamma_{1}, \gamma_{2}\right) / S_{0}^{\prime}\left(-\gamma_{1},-\gamma_{2}\right),=1
$$

by (6.9). But since, by the results of Sect. $5, \varepsilon^{\prime}$ is determined up to gauge transformation by $\mathrm{S}^{\prime}$, it must be gauge equivalent to unity, i.e.

$$
\varepsilon^{\prime}\left(\gamma_{1}, \gamma_{2}\right)=v\left(\gamma_{1}\right) v\left(\gamma_{2}\right) / v\left(\gamma_{1}+\gamma_{2}\right)
$$

for some function $v(\gamma)$. If we make a gauge transformation $u(\gamma)$ on $\varepsilon_{0}^{\prime}$, satisfying (5.23), it induces a gauge transformation given by $v(\gamma)=u(\gamma)^{2}$. Thus we may arrange that

$$
\varepsilon_{0}^{\prime}\left(\gamma_{1}, \gamma_{2}\right)=\varepsilon_{0}^{\prime}\left(-\gamma_{1},-\gamma_{2}\right)
$$

by a suitable gauge choice, and henceforth we shall assume that this has been done. [Alternatively but equivalently we could replace (6.15) by

$$
\Psi_{\Omega}(z)=\frac{1}{\sqrt{2}}\left\{u(\gamma) \Psi_{\gamma}(z)+u(-\gamma) \Psi_{-\gamma}(z)\right\},
$$

effectively fixing the relative phases of $\Psi_{\gamma}$ and $\Psi_{-\gamma}$.] This choice of cocycle takes values amongst the eighth roots of unity, the powers of $e^{i \pi / 4}$.

It is now straightforward to check that Eqs. (4.17)-(4.20) hold with

$$
\Sigma\left(\Omega_{1}, \Omega_{2}\right)=S_{0}^{\prime}\left(\gamma_{1}, \gamma_{2}\right), \quad \text { for } \quad \gamma_{1} \cdot \gamma_{2}=0,
$$

and

$$
\eta_{ \pm}\left(\Omega_{1}, \Omega_{2}\right)=\varepsilon_{0}^{\prime}\left(\gamma_{1}, \gamma_{2}\right), \quad \text { for } \quad \gamma_{1} \cdot \gamma_{2}= \pm \frac{1}{2}
$$

where $\gamma_{i} \in \Omega_{i}$. [The consistency of these equations is guaranteed by Eqs. (6.9) and (6.19).] The necessary properties (5.31) follow from (6.10), because, if $e_{1} \cdot e_{2}=0$,

$$
\Sigma\left(\Omega_{1}, \Omega_{2}\right)=S_{0}\left(e_{1}, e_{2}\right)
$$

and

$$
S_{0}\left(e_{1}, e_{2}\right)^{2}=S_{0}\left(2 e_{1}, e_{2}\right)=(-1)^{2 e_{1} \cdot e_{2}}=(-1)^{2 e_{1} \cdot e_{2}}=1 \text {, }
$$

whilst, if $e_{1} \cdot e_{2}=\frac{1}{2}$,

$$
\Sigma_{ \pm}\left(\Omega_{1}, \Omega_{2}\right)=S_{0}\left(e_{1}, \pm e_{2}\right)
$$

so that

$$
\begin{aligned}
S_{0}\left(e_{1}, e_{2}\right) \Sigma_{ \pm}\left(\Omega_{1}, \Omega_{2}\right) & =S_{0}\left(e_{1}, e_{2} \pm e_{2}\right), \\
(-1)^{e_{1} \cdot\left(e_{2} \pm e_{2}\right)} & =(-1)^{e_{1} \cdot e_{2} \pm 1 / 2},
\end{aligned}
$$

and similarly for $e_{1} e_{2}=-\frac{1}{2}$. 
To complete the construction it remains to define more carefully the space in which the vertex operators (4.4) to (4.6) act. It can not be simply $\mathscr{H} \otimes \mathscr{H}^{\prime}$, where $\mathscr{H}$ is the bosonic Fock space defined in Sect. 2 for the vertex operator construction for $g_{L}$, and $\mathscr{H}^{\prime}$ the space defined in this section for the fermions $\Psi_{\Omega}$, because the vertex operators (4.6) would not have expansions in integral powers when acting on all the states in this space. The problem is that $U(e, z), e \in \Phi_{S}$, acting on a state with momentum $x$, behaves like a Ramond field if $e, x \in \mathbb{Z}+\frac{1}{2}$, and a Neveu-Schwartz field if $e \cdot x \in \mathbb{Z}$. Similarly $\Psi_{\Omega}$ is an R field on states with momentum $y$ if $y \cdot \gamma \in \mathbb{Z}+\frac{1}{2}$, $\gamma \in \Omega^{\prime}$, whilst it is an NS field if $y \cdot \gamma \in \mathbb{Z}$. We correct for this by arranging the momentum in the $\mathscr{H}$ and $\mathscr{H}^{\prime}$ factors to be correlated so that we always have a product of R fields or a product of NS fields, thus giving an expansion in integral powers of $z$.

To correlate the momenta in this way, we divide $\mathscr{H}$ into a direct sum of subspaces $\mathscr{H}_{a}$, each consisting of states with momenta in a coset $\Lambda_{a}$ of $\Lambda_{R}(g) / \Lambda_{R}\left(g_{L}\right), 0 \leqq a \leqq 2^{n}-1$ and similarly divide $\mathscr{H}^{\prime}$ into subspaces $\mathscr{H}_{a}^{\prime}$ with momenta in $\Lambda_{a^{\prime}}^{\prime}$ the cosets of $\Lambda_{n} / 2 \Lambda_{n}$. The labelling is to be arranged so that sending $\Lambda_{a} ; \Lambda_{a}^{\prime}$ defines an isomorphism

$$
\Lambda_{R}(g) / \Lambda_{R}\left(g_{L}\right)=\Lambda_{n} / 2 \Lambda_{n}=\left(\mathbb{Z}_{2}\right)^{n}
$$

[that is, if $\Lambda_{a}+\Lambda_{b}=\Lambda_{c}$ then $\Lambda_{a}^{\prime}+\Lambda_{b}^{\prime}=\Lambda_{c}^{\prime}$ ], and so that if an orbit $\Omega_{a} \subset \Lambda_{a}$, then the corresponding orbit $\Omega_{a}^{\prime} \subset \Lambda_{a}^{\prime}$. Then we use as our space of states

$$
\bigoplus_{a}\left(\mathscr{H}_{a} \cong \mathscr{H}_{a}^{\prime}\right)
$$

and this ensures that all the vertex operators are single valued.

There are other ways of assigning momenta. Suppose $\mu_{1}, \ldots, \mu_{r}$ are fundamental weights of $g$ corresponding to the basis of simple roots of (6.6), so that

$$
2 \alpha_{i} \cdot \mu_{j} / \alpha_{i}^{2}=\delta_{i j}, \quad 1 \leqq i, j \leqq r,
$$

and $\mu_{1}^{\prime}, \ldots, \mu_{n}^{\prime}$ correspond in a similar way to $\alpha_{1}^{\prime}, \ldots, \alpha^{n}$, that is

$$
\alpha_{i}^{\prime} \cdot \mu_{j}^{\prime}=\frac{1}{2} \delta_{i j}, \quad 1 \leqq i, j \leqq n,
$$

Then for each $j, 1 \leqq j \leqq r$, we may use a space (6.27) where the momenta of $\mathscr{H}_{a}$ are taken from $\Lambda_{a}+\mu_{j}$ and the momenta of $\mathscr{H}_{a}^{\prime}$ are taken from $\Lambda_{a}^{\prime}+\mu_{j}^{\prime}$.

\section{Derivation of the Construction Using Diagram Foldings}

Each non-simply laced algebra $g$ occurs as the subalgebra of a simply-laced algebra $g_{0}$ in such a way that a level 1 representation of $\hat{g}_{0}$ provides a level 1 of representation of $\hat{g}$, because the root length of $g_{0}$ is that of the long roots of $g$. We can define $g$ as the subalgebra of $g_{0}$ left invariant by an outer automorphism of $g$, which thus corresponds to a symmetry $\tau$ of its Dynkin diagram. These symmetries are illustrated in Fig. 1. Let us postpone the discussion of the special case of $G_{2}$ until Sect. 8. In the other cases $\tau^{2}=1$. The number of simple roots of $S_{0}$ left invariant by $\tau$ is $n$, so that rank $g=r+n$. We have labelled them $\beta_{i}, 1 \leqq i \leqq r$, and $\beta_{j}^{\prime}, 1 \leqq j \leqq n$, with

$$
\tau\left(\beta_{j}\right)=\beta_{j}^{\prime}, 1 \leqq j \leqq n ; \quad \tau\left(\beta_{i}\right)=\beta_{i}, n<i \leqq \leqq .
$$


It follows from the fact, for each simple root, either $\tau\left(\beta_{j}\right)=\beta_{j}$ or $\tau\left(\beta_{j}\right) \cdot \beta_{j}=0$ that this property holds for all the roots $\beta \in \Phi\left(g_{0}\right)$, the root system of $g_{0}$, i.e.

$$
\text { either } \tau(\beta)=\beta \quad \text { or } \quad \tau(\beta) \cdot \beta=0 \text {. }
$$

In any case it is straightforward to check this directly.

Let us now consider how $\tau$ is extended from the roots to the algebra $g_{0}$, which we suppose is written in the form

$$
\begin{aligned}
{\left[H^{i}, H^{j}\right] } & =0, & & \\
{\left[H^{i}, E^{\beta}\right] } & =\beta^{i} E^{\beta}, & & \\
{\left[E^{\beta}, E^{\beta^{\prime}}\right] } & =\varepsilon\left(\beta, \beta^{\prime}\right) E^{\beta+\beta^{\prime}}, & & \text { if } \beta+\beta^{\prime} \in \Phi\left(g_{0}\right), \\
& =\beta \cdot H, & & \text { if } \beta=-\beta^{\prime}, \\
& =0, & & \text { otherwise. }
\end{aligned}
$$

We can choose the cocycle $\varepsilon$ so that it is invariant under $\tau$

$$
\varepsilon\left(\tau(\beta), \tau\left(\beta^{\prime}\right)\right)=\varepsilon\left(\beta, \beta^{\prime}\right) .
$$

[To see this, we use the construction of (5.15) with the operators $\hat{\gamma}_{i}$ and $\hat{\gamma}_{j}^{\prime}$, corresponding to $\beta_{i}$ and $\beta_{j}^{\prime}$, respectively, in (5.14) written in the order $\hat{\gamma}_{1}, \hat{\gamma}_{1}^{\prime}, \ldots, \hat{\gamma}_{n}$, $\left.\hat{\gamma}_{n}^{\prime}, \hat{\gamma}_{n+1}, \ldots, \hat{\gamma}_{r}.\right]$ It then follows that

$$
\tau\left(E^{\beta}\right)=E^{\tau(\beta)}, \quad \tau(x) \cdot H=x \cdot \tau(H)
$$

defines an automorphism of $g_{0}$. The Cartan Subalgebra of $g$ is spanned by $y \cdot H$, where $y=\tau(y)$, and in this case

$$
\left[y \cdot H, E^{\beta}+E^{\tau(\beta)}\right]=y \cdot\left\{\frac{1}{2} \beta+\frac{1}{2} \tau(\beta)\right\}\left(E^{\beta}+E^{\tau(\beta)}\right) .
$$

This $g_{0}$ has long and short roots given by

$$
\Phi_{L}=\{\beta: \beta=\tau(\beta)\}
$$

and

$$
\Phi_{S}=\left\{\frac{1}{2} \beta+\frac{1}{2} \tau(\beta): \beta \cdot \tau(\beta)=0\right\}
$$

These corresponding to step operators $E^{\beta}$ for long roots and $\frac{1}{\sqrt{2}} E^{\beta}+\frac{1}{\sqrt{2}} E^{\tau(\beta)}$ for short roots.

We now wish to reobtain the construction of Sect. 4 and 6 from this embedding. To do this we must be able to rewrite

$$
E^{\beta}(z)+E^{\tau(\beta)}(z)=U(\beta, z) c_{\beta}+U(\tau(\beta), z) c_{\tau(\beta)}
$$

as a product of functions of $\frac{1}{2} \beta+\frac{1}{2} \tau(\beta)$ and $\frac{1}{2} \beta-\frac{1}{2} \tau(\beta)$, if $\beta \cdot \tau(\beta)=0$. This is straightforward for the vertex operators:

$$
U(\beta, z)=U\left(\frac{1}{2} \beta+\frac{1}{2} \tau(\beta), z\right) U\left(\frac{1}{2} \beta-\frac{1}{2} \tau(\beta), z\right)
$$

as $\frac{1}{2} \beta+\frac{1}{2} \tau(\beta)$ and $\frac{1}{2} \beta-\frac{1}{2} \tau(\beta)$ are orthogonal, but we must also be able to factor $\hat{c}_{\beta}$ in this way. For this, we must extend the definition of $\hat{c}_{\gamma}$ from $\gamma \in \Lambda_{R}\left(g_{0}\right)$ to the 
(1)
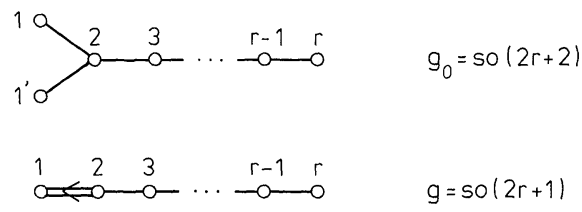

(11)

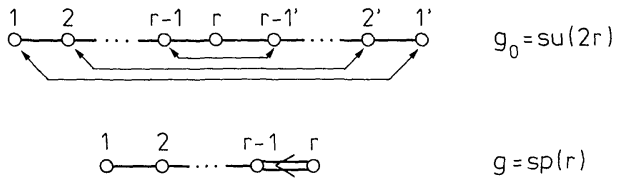

(iii)
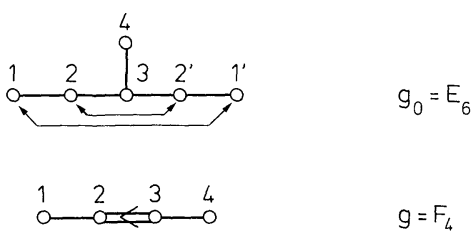

(iv)

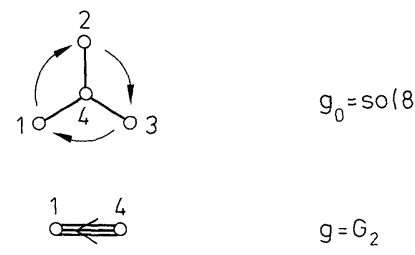

Fig. 1. Definition of non-simply-laced algebras by symmetries of simply-laced algebras

lattice spanned by $\frac{1}{2} \gamma \pm \frac{1}{2} \tau(\gamma), \gamma \in \Lambda_{R}\left(g_{0}\right)$. This lattice is isomorphic to the direct sum $\Lambda_{R}\left(g_{L}\right) \oplus \Lambda_{n}$ and we can construct a cocycle for it by taking the product of the cocycles $\varepsilon_{0}$ for $\Lambda_{R}\left(g_{L}\right)$ and $\varepsilon_{0}^{\prime}$ for $\Lambda_{n}$ used in Sects. 4-6.

$$
\varepsilon\left(\gamma_{1}, \gamma_{2}\right)=\varepsilon_{0}\left(\frac{1}{2} \gamma_{1}^{+}, \frac{1}{2} \gamma_{2}^{+}\right) \varepsilon_{0}^{\prime}\left(\frac{1}{2} \gamma_{1}^{-}, \frac{1}{2} \gamma_{2}^{-}\right),
$$

where $\gamma_{j}^{ \pm}=\frac{1}{2} \gamma_{j} \pm \frac{1}{2} \tau\left(\gamma_{j}\right)$. It is then the case that

$$
\hat{c}_{\beta}=\hat{c}_{\beta}-\hat{c}_{\beta^{+}}
$$

for $\beta^{ \pm}=\frac{1}{2} \beta \pm \frac{1}{2} \tau(\beta)$, and so

$$
\frac{1}{\sqrt{2}} E^{\beta}(z)+\frac{1}{\sqrt{2}} E^{\tau(\beta)}(z)=U\left(\beta^{+}, z\right) c_{\beta^{+}} \Psi_{\beta^{-}},
$$

where

$$
\Psi_{\beta^{-}}=\frac{1}{\sqrt{2}} U\left(\beta^{-}, z\right) c_{\beta^{-}}+\frac{1}{\sqrt{2}} U\left(-\beta^{-}, z\right) c_{-\beta^{-}} .
$$


To complete the connection with the construction of Sect. 4-6 we need to show that $\Psi_{\beta}$ - depends only on the orbit of $\frac{1}{2} \beta+\frac{1}{2} \tau(\beta)$ under the Weyl group generated by $\sigma_{\alpha}$, where $\alpha \in \Phi_{L}$. If $\beta^{+}=\gamma^{+}$, with $\beta \cdot \tau(\beta)=\gamma \cdot \tau(\gamma)=0$, then

$$
\beta-\tau(\beta)=\gamma-\tau(\gamma) \text {, }
$$

so that

$$
2=\beta \cdot \gamma-\beta \cdot \tau(\gamma),
$$

and thus either (i) $\beta \cdot \gamma=2, \beta \cdot \tau(\gamma)=0$, in which case $\beta=\gamma$ and $\beta^{-}=\gamma^{-}$; or (ii) $\beta \cdot \gamma=1, \beta \cdot \tau(\gamma)=-1$, in which case $\beta=\sigma_{\alpha}(\gamma)$ for $\alpha=\gamma-\beta$ and $\alpha \in \Phi_{L}$; or (iii) $\beta \cdot \gamma=0, \beta \cdot \tau(\gamma)=-2$, in which case $\beta=-\tau(\gamma)$ so that $\beta^{-}=\gamma^{-}$. Now, if $\alpha \in \Phi_{L}, \sigma_{\alpha}(\beta)=\beta-(\alpha \cdot \beta) \alpha$ so that $\sigma_{\alpha}\left(\beta^{-}\right)=\beta^{-}$, which shows that $\Psi_{\beta^{-}}(z)$ does indeed only depend on its orbit under $W\left(g_{L}\right)$.

\section{The Construction for $G_{2}$}

The algebra $G_{2}$ is the only simple algebra with roots of squared lengths in the ratio $3: 1$. We take the long roots $\Phi_{L}$ to be of $g$ squared length 2 , as usual, so that the short roots $\Phi_{S}$ are of squared length two thirds. The root diagram of $G_{2}$ is shown in Fig. 2 . We see that $\Phi_{L}$ is the root system of $g_{L}=\operatorname{su}(3)$, that

$$
\Lambda_{k}(g) / \Lambda_{k}\left(g_{L}\right)=\mathbb{Z}_{3},
$$

replacing (4.15), and that the elements of $\Phi_{S}$ correspond to the 3 and $\overline{3}$ representation of su (3). These correspond to the two orbits of $\Phi_{S}$ under $W\left(g_{L}\right)$ and we shall label them $\Omega_{q}$ and $\Omega_{\tilde{q}}$, respectively, so that $\Omega_{q}=\left\{\gamma_{1}, \gamma_{2}, \gamma_{3}\right\}$, in the notation of Fig. 2, and $\Omega_{\bar{q}}=\left\{-\gamma_{1},-\gamma_{2},-\gamma_{3}\right\}$.

The algebra $\widehat{G}_{2}$ has a basis consisting of $H_{m}^{j}, E_{m}^{\alpha}, E_{m}^{\gamma}, j=1,2, \alpha \in \Phi_{L}, \gamma \in \Phi_{S}$, $m \in \mathbb{Z}$; and, for a level 1 representation, the algebra is given by Eqs. (2.24) together with

$$
\begin{aligned}
{\left[H_{m}^{i}, E_{n}^{\gamma}\right] } & =\gamma^{i} E_{m+n}^{\gamma}, & & \\
{\left[E_{m}^{\alpha}, E_{n}^{\gamma}\right] } & =\varepsilon(\alpha, \gamma) E_{m+n}^{\alpha+\gamma}, & & \alpha+\gamma \in \Phi_{S} \\
& =0, & & \text { otherwise, } \\
{\left[E_{m}^{\gamma}, E_{n}^{\xi}\right] } & =\varepsilon(\gamma, \xi) E_{m+n}^{\gamma+\xi}, & & \gamma+\xi \in \Phi_{L} \\
& =\frac{2}{\sqrt{3}} \varepsilon(\gamma, \xi) E_{m+n}^{\gamma+\xi}, & & \gamma+\xi \in \Phi_{S} \\
& =e \cdot H_{m+n}-\delta_{m+n, o}, & & \gamma=-\xi \\
& =0, & & \text { otherwise. }
\end{aligned}
$$

Here again each $\varepsilon(\lambda, \mu), \lambda, \mu \in \Phi$, is \pm 1 . [In a Chevalley basis related to this CartanWeyl basis by $F^{\gamma}=\left(2 / \gamma^{2}\right)^{1 / 2} E^{\gamma}$, the structure constants would be integral.]

As in Eqs. (4.4) and (4.5), we use the usual vertex operator construction for $H_{n}^{i}$, $E^{\alpha}, i=1,2, \alpha \in \Phi_{L}, n \in \mathbb{Z}$, and for the short roots we introduce

$$
\begin{aligned}
E^{\gamma}(z) & =U(\gamma, z) c_{\gamma} \Psi_{q}(z), \\
E^{-\gamma}(z) & =U(-\gamma, z) c_{\gamma} \Psi_{\tilde{q}}(z),
\end{aligned}
$$




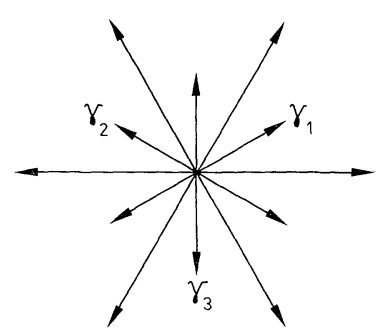

Fig. 2. The root of diagram for $G_{2}$

for $\gamma \in \Omega_{q}$ so that $-\gamma \in \Omega_{\bar{q}}$. We further need, to obtain the correct hermiticity properties,

$$
\Psi_{q}(z)^{\dagger}=\Psi_{\bar{q}}\left(1 / z^{*}\right) .
$$

This time the definition of the cocycle is easier because,

$$
(-1)^{\alpha \cdot \beta}=(-1)^{3 \alpha \cdot \beta},
$$

if either $\alpha$ or $\beta \in \Phi_{L}$ and the latter expression has values \pm 1 on the whole of $\Lambda_{k}\left(G_{L}\right)$. Thus, following the general construction of Sect. 5 , we introduce a cocycle $\hat{c}_{\gamma}$, defined on this root lattice with symmetry factor $S(\alpha, \beta)$ given by (8.6). The properties we demand of the fields $\Psi_{q}(z), \Psi_{\bar{q}}(z)$, which are not really fermions, are consequently simpler than Eqs. (4.17)-(4.19). In view of Eq. (3.1), for $e^{2}=f^{2}=2 / 3$, we need

$$
\begin{aligned}
& \Psi_{q}(z) \Psi_{q}(\zeta)=(z-\zeta)^{-2 / 3} K_{q q}(z, \zeta), \quad|\zeta|<|z|, \\
& \Psi_{q}(z) \Psi_{\bar{q}}(\zeta)=(z-\zeta)^{-4 / 3} K_{q \bar{q}}(z, \zeta), \quad|\zeta|<|z|,
\end{aligned}
$$

with analogous equations for $K_{\bar{q} q}$, and

$$
\begin{aligned}
& K_{q q}(z, \zeta)=\frac{2}{\sqrt{3}} z^{2 / 3} \Psi_{\bar{q}}(z)\left\{1+O(z-\zeta)^{2}\right\}, \\
& K_{q \bar{q}}(z, \zeta)=K_{\bar{q} q}(\zeta, z)=z^{4 / 3}\left\{1+O(z-\zeta)^{2}\right\}, \\
& K_{\bar{q} \bar{q}}(z, \zeta)=\frac{2}{\sqrt{3}} z^{2 / 3} \Psi_{q}(z)\left\{1+O(z-\zeta)^{2}\right\}
\end{aligned}
$$

To construct $\Psi_{q}(z), \Psi_{\bar{q}}(z)$, we use vertex operators acting in a Fock space ' , generated by annihilation and creation operators $\alpha_{m}^{\prime j}$ from states $|x\rangle, x \in \Lambda^{\prime}$ $=\sqrt{2} \Lambda_{R}\left(G_{2}\right)$. We define

$$
\Psi_{q}(z)=\frac{1}{\sqrt{3}}\left\{U\left(\sqrt{2} \gamma_{1}, z\right)+U\left(\sqrt{2} \gamma_{2}, z\right)+U\left(\sqrt{2} \gamma_{3}, z\right)\right\}
$$

and $\Psi_{\bar{q}}(z)$ is defined by Eq. (8.5) or, equivalently, by changing $\gamma_{i}$ to $-\gamma_{i}$ in Eq. (8.9). It is then straightforward to check that Eqs. (8.8) hold.

It remains to place restrictions on the momenta so that $E^{\gamma}(z)$, as defined by Eqs. (8.4), have expansions in terms of integral powers of $z$. We divide the space $\mathscr{H}$, in 
which the $H_{n}^{i}$ act, into cosets $\mathscr{H}_{0}, \mathscr{H}_{q}$, and $\mathscr{H}_{\bar{q}}$. With a similar division of $\mathscr{H}^{\prime}$ into $\mathscr{H}_{0}^{\prime}, \mathscr{H}_{q}^{\prime}$, and $\mathscr{H}_{q}^{\prime}$, the space we should use is

$$
\left(\mathscr{H}_{0} \otimes \mathscr{H}_{0}^{\prime}\right) \oplus\left(\mathscr{H}_{q} \otimes \mathscr{H}_{q}^{\prime}\right) \oplus\left(\mathscr{H}_{\bar{q}} \otimes \mathscr{H}_{q}^{\prime}\right) .
$$

We can obtain this construction from the embedding of $G_{2}$ in $D_{4}=\operatorname{so}(8)$ illustrated in Fig. 1 (iv). We denote the simple roots of $g_{0}=D_{4}$ by

$$
\alpha_{1}=\xi_{1}-\xi_{2}, \quad \alpha_{2}=\xi_{3}+\xi_{3}, \quad \alpha_{3}=\xi_{3}-\xi_{4}, \alpha_{4}=\xi_{2}-\xi_{3},
$$

where $\xi_{i}, 1 \leqq i \leqq 4$, is an orthonormal basis for $\mathbb{R}^{4}$, and define an outer automorphism $\tau$ of $D_{4}$ of order 3 by

$$
\tau\left(\alpha_{1}\right)=\alpha_{2}, \quad \tau\left(\alpha_{2}\right)=\alpha_{3}, \quad \tau\left(\alpha_{3}\right)=\alpha_{1}, \quad \tau\left(\alpha_{4}\right)=\alpha_{4} .
$$

Note that $\tau$ has the property that

$$
\beta+\tau(\beta) \in 2 \Lambda_{W}\left(g_{0}\right),
$$

for $\beta=\alpha_{i}, 1 \leqq i \leqq 4$, and so for any $\beta \in \Lambda_{R}\left(g_{0}\right)$.

The root system of $g=G_{2}$ is obtained from that of $g_{0}=D_{4}$ by taking the vectors

$$
\bar{\alpha}=\frac{1}{3} \alpha+\frac{1}{3} \tau(\alpha)+\frac{1}{3} \tau^{2}(\alpha), \quad \alpha \in \Phi\left(g_{0}\right) .
$$

It can be again shown that, for each $\alpha \in \Phi\left(g_{0}\right)$, either $\alpha=\tau(\alpha)$ or $\alpha \cdot \tau(\alpha)=0$, and the vectors $\bar{\alpha}$ have squared lengths 2 or $2 / 3$, respectively. Then, for $\alpha, \beta \in \Lambda_{R}\left(g_{0}\right)$,

$$
3 \bar{\alpha} \cdot \bar{\beta}=\alpha \cdot\left\{\beta+\tau(\beta)+\tau^{2}(\beta)\right\}=\alpha \cdot \beta, \quad \bmod 2,
$$

because, by (8.13), $\tau(\beta)+\tau^{2}(\beta) \in 2 \Lambda_{W}\left(g_{0}\right)$. The importance of this is that, since

$$
(-1)^{3 \bar{\alpha} \cdot \bar{\beta}}=(-1)^{\alpha \cdot \beta},
$$

we can construct a suitable cocycle for $D_{4}$ by using a function $c_{\bar{\alpha}}$ of $\bar{\alpha}$, invariant under $\tau$, because $\tau(\bar{\alpha})=\bar{\alpha}$. Then, with this cocycle,

$$
\tau\left(E^{\alpha}\right)=E^{\tau(\alpha)}, \quad \tau^{-1}(x) \cdot H=x \cdot \tau(H)
$$

defines an automorphism of $D_{4}$ and this extends to $\hat{D}_{4}$ with $\tau$ mapping

$$
E^{\alpha}(z)=U(\alpha, z) c_{\bar{\alpha}} \rightarrow U(\tau(\alpha), z) c_{\bar{\alpha}} .
$$

$\widehat{G}_{2}$ is obtained as the subalgebra left invariant by $\tau$. We take as basis the components of

$$
\alpha \cdot H(z) ; \quad E^{\alpha}(z), \quad \alpha=\tau(\alpha),
$$

and

$$
\frac{1}{\sqrt{3}}\left\{E^{\alpha}(z)+E^{\tau(\alpha)}(z)+E^{\tau^{2}(\alpha)}(z)\right\}, \quad \alpha \cdot \tau(\alpha)=0,
$$

where $\alpha \in \Phi\left(g_{0}\right)$. The latter operator can be rewritten as

$$
U(\bar{\alpha}, z) c_{\bar{\alpha}} \Psi(z)
$$

where

$$
\Psi(z)=\Psi_{q}(z) \quad \text { or } \quad \Psi_{\bar{q}}(z)
$$


with

$$
\begin{gathered}
\Psi_{q}(z)=\frac{1}{\sqrt{3}}\left\{U\left(\lambda_{1}, z\right)+U\left(\lambda_{2}, z\right)+U\left(\lambda_{3}, z\right)\right\}, \\
\lambda_{1}=\frac{2}{3}\left(\xi_{1}-\xi_{2}-\xi_{3}\right), \quad \lambda_{2}=\tau\left(\lambda_{1}\right), \quad \lambda_{3}=\tau^{2}\left(\lambda_{1}\right),
\end{gathered}
$$

and $\Psi_{\bar{q}}$ similarly defined replacing $\lambda_{i}$ by $-\lambda_{i}$. Thus we have regained our previous construction.

\section{Generalisations of the Construction, Triality, and the Magic Square}

In this section we generalise the construction of Sects. 4-6 and make a remarkable connection with Freudenthal's magic square. In Sect. 4 we saw that if $\Lambda=\Lambda_{R}(g)$ and $\Lambda_{0}=\Lambda_{R}\left(g_{L}\right)$, for $g=\operatorname{so}(2 r+1)$, sp $(r)$ or $F_{4}$, then

(a) $\Lambda_{0}$ is an even integral lattice;

(b) $\Lambda / \Lambda_{0} \equiv\left\{\Lambda_{a}: 0 \leqq a \leqq 2^{n}-1\right\} \cong\left(\mathbb{Z}_{2}\right)^{n}$;

(c) $\Lambda_{0} \cup \Lambda_{a}$ is an integral lattice;

$n$ being $1, r-1$ and 2 respectively, that is the number of short simple roots of $g$. It follows from these properties that $2 \Lambda \subset \Lambda_{0} \subset \Lambda$ and $\Lambda_{0} \subset \Lambda^{*}$, (the lattice dual to $\Lambda$ ). Hence $\sqrt{2} \Lambda$ is an integral lattice. Conversely given lattices $\Lambda_{0} \subset \Lambda$ with these properties (a), (b), and (c), it can easily be checked that $\Lambda_{0}^{(2)} \cup \Lambda^{(1)}$ satisfies (2.1) and hence constitutes the root system for a Lie algebra $g_{\Lambda}$, where $\Gamma^{(m)}$ denotes the set of points of squared length $m$ on the lattice $\Gamma$. Another example of such a system, satisfying (a), (b), and (c), is provided by $\Lambda=1 / \sqrt{2} \Lambda_{R}(\mathrm{su}(n+1))$ and $\Lambda_{0}=2 \Lambda$, though in this case $\Lambda_{0}^{(2)}$ is empty so that $g_{A}=\operatorname{su}(n+1)$ is simply-laced but with the root lengths set equal to 1 rather than $\sqrt{2}$.

These four examples have another common feature; they can be understood in terms of the folding procedure of Sect. 7. Let $\Lambda^{\prime}$ be an even integral lattice with an automorphism $\tau$ of order 2 , so that $\tau\left(\Lambda^{\prime}\right)=\Lambda^{\prime}, \tau^{2}=1$, and define

$$
\begin{aligned}
\Lambda & =\left\{\frac{1}{2} x+\frac{1}{2} \tau(x): x \in \Lambda^{\prime}\right\}, \\
\Lambda_{0} & =\left\{x: x \in \Lambda^{\prime}, \quad \tau(x)=x\right\} .
\end{aligned}
$$

Then properties (a) and (b) follow for some value of $n$, while (c) is an extra property, which is valid if $\Lambda^{\prime}=\Lambda_{R}\left(g^{\prime}\right)$ or if $\Lambda^{\prime}=\Lambda_{R}(\operatorname{su}(n+1)) \oplus \Lambda_{R}(\operatorname{su}(n+1))$ and $\tau$ interchanges the two pieces.

The cosets $\Lambda_{a}$ have a property of being either even or odd, that is, if $x \in \Lambda_{a}, x^{2}$ is either an even or an odd integer, irrespective of the choice of $x$. It is this property which determines whether $x \cdot y \in \mathbb{Z}$ or $\mathbb{Z}+\frac{1}{2}$; if $x \in \Lambda_{a}, y \in \Lambda_{b}, x \cdot y$ is an integer if and only if either none or two of $\Lambda_{a}, \Lambda_{b}, \Lambda_{a}+\Lambda_{b}$ are odd, and otherwise $x \cdot y \in \mathbb{Z}+\frac{1}{2}$.

We say that two systems satisfying (a), (b), and (c) match if they have the same value of $n$ (though possibly different dimensions) and we can establish an isomorphism between the $\left(\mathbb{Z}_{2}\right)^{n}$ groups which preserves the oddness and evenness properties. 
We now define a way of combining two matching systems $\Lambda$ and $\bar{\Lambda}$, satisfying (a), (b), and (c), to obtain an even integral lattice, which we denote by $\Lambda \cdot \bar{\Lambda}$, whose dimension is the sum of that of its components.

$$
\Lambda \cdot \bar{\Lambda} \equiv \bigcup_{a} \Lambda_{a} \oplus \bar{\Lambda}_{a}
$$

Since it is even and integral, $(\Lambda \cdot \bar{\Lambda})^{(2)}$ constitutes the root system of a simply-laced Lie algebra $g_{A \cdot \bar{A}}$. We shall now present the corresponding multiplication rules for the lattices $\Lambda_{R}\left(B_{r}\right), \Lambda_{R}\left(C_{r}\right), \Lambda_{R}\left(F_{4}\right), \Lambda_{R}\left(A_{r}\right) / \sqrt{2}$, which match when $n$ does. The values $n=1$ and 2 are special. It is easy to check that if $n=1$ the only possibilities are $\Lambda_{R}\left(B_{r}\right)$ [noting that $\Lambda_{R}\left(B_{1}\right)=\Lambda_{R}\left(A_{1}\right) / \sqrt{2}$ ] and that

$$
\Lambda_{R}\left(B_{r}\right) \cdot \Lambda_{R}\left(B_{s}\right)=\Lambda_{R}\left(D_{r+s}\right) .
$$

If $n=2$ the multiplication table giving $g_{\Lambda \cdot \bar{\Lambda}}$ is

\begin{tabular}{llll}
\hline & $\frac{1}{\sqrt{2}} \Lambda_{R}\left(A_{2}\right)$ & $\Lambda_{R}\left(C_{3}\right)$ & $\Lambda_{R}\left(F_{4}\right)$ \\
\hline$\frac{1}{\sqrt{2}} \Lambda_{R}\left(A_{2}\right)$ & $A_{2} \oplus A_{2}$ & $A_{5}$ & $E_{6}$ \\
$\Lambda_{R}\left(C_{3}\right)$ & $A_{5}$ & $D_{6}$ & $E_{7}$ \\
$\Lambda_{R}\left(F_{4}\right)$ & $E_{6}$ & $E_{7}$ & $E_{8}$ \\
\hline
\end{tabular}

For $n=N-1 \geqq 3$, we have

\begin{tabular}{llc}
\hline & $\frac{1}{\sqrt{2}} \Lambda_{R}\left(A_{N-1}\right)$ & $\Lambda_{R}\left(C_{N}\right)$ \\
\hline$\frac{1}{\sqrt{2}} \Lambda_{R}\left(A_{N-1}\right)$ & $\operatorname{su}(N) \oplus \operatorname{su}(N)$ & $\operatorname{su}(2 N)$ \\
$\Lambda_{R}\left(C_{N}\right)$ & $\operatorname{su}(2 N)$ & so $(4 N)$ \\
\hline
\end{tabular}

The table for $n=2$ bears a striking resemblence to Freudenthal's magic square and this will be enhanced when we consider the relation to our vertex operator construction.

Associated with any one of the systems $\Lambda_{0} \subset \Lambda$, satisfying (a), (b), and (c), we can define bosonic fields $H^{i}(z), 1 \leqq i \leqq n ; E^{\alpha}(z), \alpha \in \Lambda^{(2)}$, and fermionic fields $\Psi^{\gamma}(z)$, $\gamma \in \Lambda^{(1)}$, acting in a space $\mathscr{H}=\bigoplus_{a} \mathscr{H}_{a}$. We shall denote by $\mathbb{C}_{n+1}$ the system given by $g=\operatorname{su}(n+1)$, by $\mathbb{H}_{n+1}$ the system given by $g=\operatorname{sp}(n+1)$, by $\mathbb{D}_{3}$ the system given by $g=F_{4}$, and henceforth omit from consideration $g=B_{r}$ which is only relevant for $n=1$. Corresponding to the combination of lattices (9.2), we can define a bosonic combination of these fields, given two matching systems $\Lambda$ and $\bar{\Lambda}$, which will yield a 
level 1 representation of the affinised version of the simply-laced algebra $g_{A \cdot \bar{A}}$, defined by (9.2). This representation is generated by the bose fields:

$$
\begin{gathered}
H^{i}(z), \quad 1 \leqq i \leqq \operatorname{dim} \Lambda ; \quad \bar{H}^{j}(z), 1 \leqq j \leqq \operatorname{dim} \bar{\Lambda} ; \\
E^{\alpha}(z), \quad \bar{E}^{\alpha^{\prime}}(z), \quad \alpha \in \Lambda_{0}^{(2)}, \quad \alpha^{\prime} \in \bar{\Lambda}_{0}^{(2)} ; \\
\Psi^{\gamma}(z) \bar{\Psi}^{\gamma^{\prime}}(z), \quad \gamma \in \Omega_{i j}, \quad \gamma^{\prime} \in \bar{\Omega}_{i j}, \quad 1 \leqq i<j \leqq n+1 .
\end{gathered}
$$

The main construction of this paper resembles this but consists instead of multiplying the system associated with $\Lambda$ by yet another system that we shall denote $\mathbb{R}_{n}$, say, consisting of $\frac{1}{2} n(n+1)$ real fermi fields, to obtain

$$
\begin{aligned}
& H^{i}(z), \quad 1 \leqq i \leqq \operatorname{dim} \Lambda ; \quad E^{\alpha}(z), \quad \alpha \in \Lambda_{0}^{(2)} \\
& \Psi^{\gamma}(z) \Psi_{\Omega_{i j}}(z), \quad \gamma \in \Omega_{i j}, \quad 1 \leqq i<j \leqq n+1 .
\end{aligned}
$$

Reinterpreting the tables in terms of (9.4), we can augment them by an extra row and column to take account of (9.5). The resultant tables are:

\begin{tabular}{llll}
\hline & $\mathbb{R}_{N}$ & $\mathbb{C}_{N}$ & $\mathbb{H}_{N}$ \\
\hline $\mathbb{R}_{N}$ & $\mathrm{so}(N)$ & $\operatorname{su}(N)$ & $\operatorname{sp}(N)$ \\
$\mathbb{C}_{N}$ & $\operatorname{su}(N)$ & $\operatorname{su}(N) \oplus \operatorname{su}(N)$ & $\operatorname{su}(2 N)$ \\
$\mathbb{H}_{N}$ & $\operatorname{sp}(N)$ & $\operatorname{su}(2 N)$ & $\operatorname{so}(4 N)$ \\
\hline
\end{tabular}

where, as before, $N=n+1 \geqq 4$. In the special case of $n=2$, in which we can also use $\mathbb{D}_{3}$, we now obtain precisely the magic square of Freudenthal:

\begin{tabular}{lllll}
\hline & $\mathbb{R}_{3}$ & $\mathbb{C}_{3}$ & $\mathbb{H}_{3}$ & $\mathbb{O}_{3}$ \\
\hline $\mathbb{R}_{3}$ & $A_{1}$ & $A_{2}$ & $C_{3}$ & $F_{4}$ \\
$\mathbb{C}_{3}$ & $A_{2}$ & $A_{2} \oplus A_{2}$ & $A_{5}$ & $E_{6}$ \\
$\mathbb{H}_{3}$ & $C_{3}$ & $A_{5}$ & $D_{6}$ & $E_{7}$ \\
$\mathbb{O}_{3}$ & $F_{4}$ & $E_{6}$ & $E_{7}$ & $E_{8}$ \\
\hline
\end{tabular}

All of the corresponding representations of Kac-Moody algebras are at level 1 except for the su $(N)$ obtained from $\mathbb{R}_{N} \times \mathbb{C}_{N}$ and so $(N)$ obtained from $\mathbb{R}_{N} \times \mathbb{R}_{N}$, which are level 2. The $E_{8}$ entry in this last table was already constructed in this fashion in [9] and the other entries in these tables constitute the promised generalisation of that work.

The notation $\mathbb{R}, \mathbb{C}, \mathbb{H}, \mathbb{D}$ that we have used is of course suggestive of the real, complex, quaternionic and octonionic division algebras, respectively, and is justified because the real dimensions of these algebras, $1,2,4$, and 8 , respectively, coincide with the number of fermi fields associated with each coset $\Lambda / \Lambda_{0}$ in the corresponding system. These fermi fields can be used to define the appropriate multiplication law [10]. 


\section{The Virasoro Algebra}

In this section we verify directly that the Sugawara construction of the Virasoro algebra yields the anticipated values (1.6) of $c$ in (1.2). This argument generalises one due to Frenkel [10]; see [3] for a review. We shall calculate

$$
\mathscr{L}(z)=\frac{1}{\beta} \sum_{a} \underset{\times}{\times} T^{a}(z) T^{a}(z) \stackrel{\times}{\times},
$$

using the vertex operator representation of Sects. $4-6$, where

$$
\beta=\frac{2}{r}\left[r+n_{L}+(S / L)^{2} n_{S}\right]
$$

with $r=\operatorname{rank} g$. The part of the sum corresponding to the Cartan subalgebra of $g$.

$$
\sum_{a=1}^{r} \stackrel{\times}{\times} T^{a}(z) T^{a}(z) \underset{\times}{\stackrel{\times}{\times}}=\sum_{j=1}^{r}: P^{j}(z)^{2}:,
$$

where $P^{j}(z)$ is defined by Eq. (2.23). The corresponding contribution from the long roots of $g$ is

$$
\sum_{\times}^{\times} E^{\alpha}(z) E^{-\alpha}(z) \underset{\times}{\times}=\frac{1}{2} \sum:\{\alpha \cdot P(z)\}^{2}:,
$$

where the sums are over $\alpha \in \Phi_{L}$, by the same argument as in the simply-laced case (see e.g. [3]). The contribution of the short roots is

$$
\begin{aligned}
\lim _{z \rightarrow \zeta} \sum_{\Omega} \sum_{\gamma \in \Omega} \frac{\sqrt{z \zeta}}{z-\zeta} \\
\quad\left\{: \exp (i \gamma \cdot[Q(z)-Q(\zeta)]):\left[\begin{array}{ll}
\circ \\
\circ
\end{array} \Psi_{\Omega}(z) \Psi_{\Omega}(\zeta) \circ+\Delta(z, \zeta)\right]-\frac{2 \sqrt{z \zeta}}{z-\zeta}\right\},
\end{aligned}
$$

where

$$
\Delta(z, \zeta)=\frac{\sqrt{z \zeta}}{z-\zeta} \quad \text { or } \quad \frac{1}{2} \frac{z+\zeta}{z-\zeta}
$$

in the Neveu-Schwarz (NS) or Ramond case (R), respectively. We can rearrange (10.5) as $z$ tends to $\zeta$ of

$$
\begin{aligned}
& \sum_{\Omega} \sum_{\gamma \in \Omega} \frac{\sqrt{z \zeta}}{z-\zeta}: \exp (i \gamma \cdot[Q(z)-Q(\zeta)]):\left[\circ \Psi_{\Omega}(z) \Psi_{\Omega}(\zeta) \stackrel{\circ}{\circ}+\Delta(z, \zeta)-\frac{\sqrt{z \zeta}}{z-\zeta}\right] \\
& \quad+\frac{z \zeta}{(z-\zeta)^{2}} \sum_{\gamma \in \Phi_{L}}\{\cos (\gamma \cdot[Q(z)-Q(\zeta)])-1\} .
\end{aligned}
$$

Now

$$
\lim _{z \rightarrow \zeta} \frac{1}{z-\zeta} \stackrel{\circ}{\circ} \Psi_{\Omega}(z) \Psi_{\Omega}(\zeta) \stackrel{\circ}{\circ}=\circ \frac{\circ}{\circ} \frac{d \Psi_{\Omega}}{d z} \Psi_{\Omega} \circ \text { 。, }
$$

so that (10.7) yields

$$
\sum_{\Omega}|\Omega|\left\{z \stackrel{\circ}{\circ} \frac{\mathrm{d} \Psi_{\Omega}}{d z} \Psi_{\Omega} \circ+2 \varepsilon\right\}+\frac{1}{2} \sum_{\gamma \in \Phi_{S}}:\{\alpha \cdot P(z)\}^{2}:
$$


where $|\Omega|$ denotes the number of points in an orbit $\Omega$, and $\varepsilon$ is 0 in the NS case and $\frac{1}{16}$ in the $\mathrm{R}$ case. If we write

$$
L_{\Omega}(z)=\frac{1}{2} z \circ \frac{d \Psi_{\Omega}}{d z} \Psi_{\Omega} \circ+\varepsilon,
$$

each $L_{\Omega}(z)$ satisfies the Virasoro algebra with $c=\frac{1}{2}$, but they do not commute for different $\Omega$. Thus

$$
\sum_{a} \times T^{a}(z) T^{a}(z) \underset{\times}{\times}=: P(z)^{2}:+\frac{1}{2} \sum_{\alpha \in \Phi}:[\alpha \cdot P(z)]^{2}:+2|\Omega| \sum_{\Omega} L_{\Omega}(z) .
$$

To simplify this, we use

$$
\sum_{\alpha \in \Phi} \alpha \alpha^{T}=\chi I_{r}
$$

from which it follows that

$$
r \chi=2 n_{L}+2 n_{S}(S / L)^{2}
$$

and so

$$
1+\frac{1}{2} \chi=\frac{1}{2} \beta
$$

This means that we can rewrite

$$
\mathscr{L}(z)=\frac{1}{2} \sum_{j=1}^{r}: P(z)^{2}:+\frac{|\Omega|}{1+\tilde{h}} \sum_{\Omega} L_{\Omega}(z),
$$

where we have also used (1.5).

Evaluating case by case, we find that the factor

$$
\frac{|\Omega|}{1+\tilde{h}}=\frac{4}{n+3}
$$

where $n$ is again the number of short simple roots of $g$. Thus, for so $(2 r+1)$, which has $n=1$, this is unity and we have the Virasoro algebra corresponding to a single real fermion, which has $c$-number $\frac{1}{2}$ as required. In general the $c$-number associated with

$$
\frac{4}{h+3} \sum_{\Omega} L_{\Omega}(z)
$$

is

$$
\frac{1}{2} \frac{4}{n+3} \frac{1}{2} n(n+1)=\frac{n(n+1)}{n+3}
$$

as only the diagonal terms contribute and there are $\frac{1}{2} n(n+1)$ of these. This agrees with (1.6) and (1.11). We can verify directly that (10.17) stisfies the Virasoro algebra, by using

$$
L_{\Omega}(z)=\frac{1}{2}:\{\gamma \cdot P(z)\}^{2}:+\frac{1}{4} U^{\prime}(2 \gamma, z) c_{\gamma}^{\prime 2}+\frac{1}{4} U^{\prime}(-2 \gamma, z) c_{-\gamma}^{\prime 2},
$$

where $\gamma \in \Omega^{\prime}$ and the notation is as in Sect. 6 . 


\section{Conclusion}

In this section we want to mention our reasons for thinking that the nature of the construction we have found is relevant to a better understanding of the fermionic string theory or possibly, superstring theory.

The Kac-Frenkel-Segal construction using tachyon vertex operators furnished level 1 representations of affinisations of simply-laced Lie algebras. It was pointed out by Goddard and Olive [4] and by Frenkel [12] that a covariant version of this construction could be applied to "Lorentzian algebras" corresponding to even integral lattices in twenty six dimensions or less. The even self-dual lattice in twenty six dimensions, $\mathrm{II}^{25,1}$ related, it was conjectured, to the 26-dimensional theory of open bosonic strings.

There is a corresponding algebra $\mathrm{II}^{9,1}$ for a ten-dimensional even self-dual lattice and its Dynkin diagram is $E_{10}$, the natural extension of those for the $E$ series of Lie algebras that have been proposed as the symmetry of 11-dimensional supergravity theory when compactified to one dimension [13]. However, $E_{10}$ would seem to be an inappropriate Dynkin diagram for the fermionic string theory in 10 dimensions for two related reasons. First, the algebra $\mathrm{II}^{9,1}$ can be represented by bosonic tachyonic vertex operators the critical dimension of whose Virasoro algebra is 26 , not 10 . Second, the fermionic string theory possesses two tachyon states with mass squared -2 and -1 respectively, rather as if it corresponded to a non-simply laced algebra. The first tachyon actually decouples but its Regge recurrences are physical. The second tachyon corresponding to the "short roots" has an emission vertex proportional to $: \exp (i k \cdot Q):$ with $k^{2}=1$, times a fermionic field, the Neveu-Schwarz field. This therefore resembles the construction of our paper when the ratio of the squared length of the roots to the short roots is 2 . We therefore want a non-simply laced Dynkin diagram with 10 points, which can presumably be obtained by our folding procedure from a simply-laced diagram. An example of what we mean, though we are uncertain that it is the correct example, is provided by the Dynkin diagram of the Lorentzian algebra corresponding to the 18-dimensional even self-dual lattice $I I^{17.1}$. Its diagram has 19 points and just one $\mathbb{Z}_{2}$ symmetry:

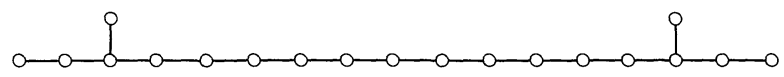

Folding the diagram, identifying points related by the symmetry, leads to a nonsimply laced diagram with 10 points as described:

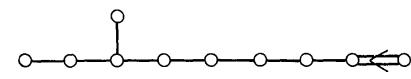

Our suspicion is that this or a similar diagram, perhaps with the arrow reversed, is relevant to the fermionic string theory. To substantiate this we must repeat our analysis in the Lorentzian situation. This is why we tried to couch our arguments in as general a form as possible.

If we could generalize our procedure in this way we would have understood how the fermionic string theory could be obtained from a bosonic string theory in a higher number of dimensions. It would be more statisfactory if the higher number of dimensions were 26 rather than 18 , but the $\mathrm{II}^{25,1}$ Dynkin diagram possesses an infinite number of points so that an infinite order symmetry would be needed. 
Another point of potential relevance to super or heterotic string theory that we should like to point out occurs in Sect. 9 where we reveal a connection between octonions, $F_{4}$, and $E_{8}$, and hence with $\left(F_{4}\right)_{L}=D_{4}$.

Finally let us mention that the representations obtained for the $\mathrm{sp}(n)$ algebra allow a realization of the FQS [7] discrete series of the Virasoro algebra in terms of bosonic variables.

Goddard, Kent and Olive [14] gave a construction of the FQS series using the Sugawara construction for the $\mathrm{sp}(n) / \mathrm{sp}(n-1) \times \mathrm{sp}(1)$ coset. Using formulae (4.4) and (4.6) for the $\operatorname{sp}(n)$ generators in the expressions of [14] the contributions coming from the simply-laced part cancel out leaving only the contributions originating in the $\Psi_{\Omega}$ fields. Using the explicit expressions for $\Psi_{\Omega},(6.11)$ and (6.15), it is possible to write the Virasoro generators for $c<1$ purely in terms of bosonic fields.

\section{References}

1. Frenkel, I. B., Kac, V.G.: Invent. Math. 62, 23 (1980)

2. Segal, G.: Unitary representations of some infinite dimensional groups. Commun. Math. Phys. 80, 301 (1981)

3. Goddard, P., Olive, D.: Kac-Moody and Virasoro algebras in relation to quantum physics. Int. J. Mod. Phys. A (to be published)

4. Goddard, P., Olive, D.: In: Vertex operators in mathematics and physics. Lepowsky, J. et al. (eds.) MSRI Publication No. 3. Berlin, Heidelberg, New York: Springer 1984, p. 51

5. Gross, D., Harvey, J., Martinec, E., Rohm, R.: Heterotic string. Phys. Rev. Lett. 54, 502 (1985): Heterotic string theory. (I). The free heterotic string. Nucl. Phys. B256, 253 (1985)

6. Goddard, P., Olive, D.: Kac-Moody algebras, conformal symmetry and critical exponents. Nucl. Phys. B257 [FS 14], 226 (1985)

7. Friedan, D., Qiu, Z., Shenker, S.: Conformal invariance, unitarity, and critical exponents in two dimensions. Phys. Rev. Lett. 52, 1575 (1984)

8. Lepowsky, J., Primc, M.: In: Vertex operators in mathematics and physics. Lepowsky, J. et al. (eds.) MSRI Publication No.3. Berlin, Heidelberg, New York: Springer 1984, p. 143 Alvarez, O., Mangano, M., Windey, P.: Berkeley preprint

9. Goddard, P., Olive, D., Schwimmer, A.: The heterotic string and a fermionic construction of the $E_{8}$ Kac-Moody algebra. Phys. Lett. 157B, 393 (1985)

10. Goddard, P., Nahm, W., Olive, D., Schwimmer, A.: To appear

11. Frenkel, I.: J. Funct. Anal. 44, 259 (1981)

12. Frenkel, I.: Lectures in Applied Mathematics 21, 325 (1985)

13. Julia, B.: In: Vertex operators in mathematics and physics. Lepowsky, J. et al. (eds.) MSRI Publication No. 3. Berlin, Heidelberg, New York: Springer 1984, p. 393

14. Goddard, P., Kent, A., Olive, D.: Phys. Lett. 152B, 88 (1985)

15. Bais, A., Englert, F., Taormina, A., Zizzi, P.: Preprint

Communicated by A. Jaffe

Received July 14, 1986

Note added in proof. In the course of the preparation of this paper, we became aware of other work on non-simply-laced algebras. A paper by A. Bais, F. Englert, A. Taormina, and P. Zizzi [15] deals with representations with level higher than 1 , such that the Virasoro $c$-number is an integer, and does not involve the use of fermion fields. Thus it does not appear to be directly related to our present work. On the other hand, work to appear by D. Bernard and J. Thierry-Mieg has a considerable overlap with that presented here, and we are grateful to them for comparisons between our approaches. 